\title{
Evaluation of statistical climate reconstruction methods based on pseudoproxy experiments using linear and machine learning methods
}

\author{
Zeguo Zhang, Sebastian Wagner, Marlene Klockmann, Eduardo Zorita \\ Helmholtz Zentrum Hereon, Institute of Coastal Systems, 21502 Geesthacht, Germany \\ 5 Correspondence to: Zeguo Zhang (zeguo.zhang@ @ereon.de)
}

\begin{abstract}
Three different climate field reconstruction (CFR) methods employed to reconstruct North Atlantic-European (NAE) and Northern Hemisphere (NH) summer season temperature over the past millennium from proxy records are tested in the framework of pseudoproxy experiments derived from three climate simulations with Earth System Models. Two of these methods are traditional multivariate linear methods (Principal Components Regression, PCR and Canonical Correlation

10 Analysis, CCA), whereas the third method (Bidirectional Long-Short-Term Memory Neural Network, Bi-LSTM) belongs to the category of machine learning methods. The Bi-LSTM method does not need to assume linear and temporally stable relationships between the underlying proxy network and the targeted climate field, in contrast to PCR and CCA. In addition, Bi-LSTM incorporates information on the serial correlation of the time series. All three methods tested herein achieve reasonable reconstruction performance in both spatial and temporal scale. Generally, the reconstruction skill is higher in

15 regions with denser proxy coverage, but reconstruction skill is also achieved in proxy-free areas due to climate teleconnections. All three CFR methodologies generally tend to more strongly underestimate the target temperature variations as more noise is introduced into the pseudoproxies. The Bi-LSTM method tested in our experiments shows relatively worse reconstruction skills compared to PCR and CCA, yet it brings some encouraging results on capturing extreme cooling climate signals. This indicates that this nonlinear CFR method could be a potential methodology for past climate extremes analysis.
\end{abstract}

\section{Introduction}

The reconstruction of past climates helps to better understand past climate variability and pose the projected future climate evolution against the backdrop of natural climate variability (PAGES 2k Consortium, 2013, 2017, 2019; PAGES Hydro2k Consortium, 2017; Schmidt, 2010; Evans et al., 2014; Christiansen and Ljungqvist, 2016). Paleoclimate reconstruction also provide us with a deeper perspective to better understand the effect of external forcing on climate (Smerdon et al., 2011, 2016;

25 Smerdon, 2012; Wang et al., 2017). However, systematic observational/instrumental climate records are only available starting from the middle of the 19th century, which hinders to capture the full spectrum of past climate variations. Consequently, our understanding of climate variations in earlier centuries is mainly based on indirect proxy records (such as tree rings, ice cores), etc. 
https://doi.org/10.5194/cp-2022-5

Preprint. Discussion started: 31 January 2022

(c) Author(s) 2022. CC BY 4.0 License.

(c) (i)

The reconstruction of past climates based on proxy data requires the application of statistical methods to translate the information contained in the proxy records into climate variables such as temperature. These methods add an additional layer of statistical uncertainty and bias to the final reconstruction, in addition to the uncertainties originating in the sparse data coverage and in the presence of non-climatic variability in the proxy records. All these sources of error impact the quality of climate reconstructions. One way to estimate this impact is the test of reconstruction methods in the controlled conditions provided by climate simulations with state-of-the-art Earth System models. These models provide virtual climate trajectories, which although possibly not completely realistic, are from the model's perspective physically consistent. The skill of the statistical method, the impact of proxy network coverage and of the amount of climate signal present in the proxy records can thus be evaluated in that virtual reality of climate models, once adequate synthetic proxy records are constructed. These tests are generally denoted pseudo-proxy experiments (PPEs, Smerdon, 2012, Gómez-Navarro et al, 2017).

Many scientific studies that employ pseudo-proxies and real proxies have focused on global and hemisphere climate field or climate index reconstructions (Mann et al., 2002, 2005; von Storch et al., 2004; Smerdon, 2012; Michel et al., 2020; Hernández et al., 2020). These studies have identified several deficiencies that are common to most climate reconstructions methods, such as a general tendency to 'regress to the mean', which results in an underestimation of the reconstructed climate variability. This underestimation becomes more evident when the available proxy information becomes of less quality - diminishing the climate signal contained in the proxy records. In addition, sparser networks - shrinking proxy network coverage - may lead to biased reconstructions (Wang et al., 2014; Evans et al., 2014; Amrhein et al., 2020; Po-Chedley et al., 2020). Thus, significant scope still remains for further developing and evaluating CFR methodologies and in designing methods that are less prone to those common deficiencies (Christiansen and Ljungqvist, 2016).

In the present study, we test a new non-linear CFR method that belongs to the machine learning family, a Bidirectional LongShort-Term Neural Network (Bi-LSTM). We compare the performance of this method with two well-established classical multi-variate linear regression methods, Principal Component Regression (PCR) and Canonical Correlation Analysis (CCA). Traditional CFRs usually assume linear and temporally stable relationships between the local variables captured by the proxy network and the target climate field. Likewise, the spatial patterns of climate variability are considered as stationary (Pyrina et al., 2017; Wang et al., 2014; Smerdon et al., 2016). However, climate change is dynamic and chaotic, and many links between climate fields can be non-linear (Schneider et al., 2018; Dueben and Bauer, 2018; Huntingford et al., 2019; Nadiga,

55 2020). Nonlinear machine leaning-based CFR methods (for instance, Artificial Neural Networks-ANN) could help capture capture underlying linear and nonlinear relationships between proxy records and the large-scale climate as realistically as possible (Rasp and Lerch, 2018; Schneider et al., 2018; Rolnick et al., 2019; Huang et al., 2020; Nadiga, 2020; Chattopadhyay et al., 2020; Lindgren et al., 2021). Moreover, machine-learning methods do not necessarily rely on statistical methods to first obtain the principal spatial climate patterns, such as Principal Component Analysis-PCA. The full inherent variability in the

60 original dataset is sequentially and dynamically adjusted and captured with optimized hyper-parameters during the model training process (Goodfellow et al., 2016). The classical recurrent neural network (RNN) and Long Short-Term Memory Network (LSTM) can usually only receive and process information from prior forward inference steps, whereas the Bi-LSTM 
handles information from both forward and backward temporal directions (Graves and Schmidhuber, 2005). It has been demonstrated that the Bi-LSTM model can achieve better performance for some classification and prediction tasks (Su et al.,

65 2021; Biswas and Sinha, 2021; Biswas et al., 2021). Since climate dynamics usually exhibit temporal dependencies, the BiLSTM method might learn these dependencies better, which can provide another advantage to capture the time evolution of the reconstructed climate field. To our knowledge, Bi-LSTM method is applied for the first time in the context of paleo CFRs. In this evaluation of climate reconstruction methods, we focus on the whole Northern Hemisphere temperature field and on the temperature field of the North Atlantic European region. In the North Atlantic region, the most important mode of temperature variations at longer time series is the Atlantic Multidecadal Variability (AMV). The index of the AMV is defined as decadal filtered surface temperature anomaly over North Atlantic regions $\left(95^{\circ} \mathrm{W}-30^{\circ} \mathrm{E}, 0-70^{\circ} \mathrm{N}\right.$, excluding the Mediterranean and Hudson Bay following Knight et al., 2006). It has been shown that AMV is related to many prominent examples of regional or even hemispheric multidecadal climate variability, for example European and North America summer climate variability (Knight et al., 2006; Qasmi et al., 2017). In this context, we test the reconstruction skill for the spatial resolved summer seasonal temperature anomalies over NH and NAE, as well as for the spatially averaged AMV and NH summer temperature anomalies, calculated from the spatially resolved reconstructed fields. The reconstruction of mean temperature series could provide a general assessment of the skill to reconstruct extreme temperature phases (e.g. related to volcanic eruptions or changes in solar activity) serving as benchmarks to test the potential capability of different CFR methods on those anomalies.

80 Regarding the networks of real proxies used so far, St. George and Esper (2019) reviewed contemporary studies on previous NH temperature reconstructions based on tree ring proxies (Mann et al., 1998, 2008, 2007, 2009a, 2009b; PAGES2k Consortium). St. George and Esper concluded that the present-day generation of tree ring proxy based reconstructions exhibit high correlations with seasonal hemispheric summer temperatures and display relatively better skills in tracking year-to-year climatic variabilities and decadal fluctuations than former proxy networks, as found by Wilson et al., (2016) and Anchukaitis 85 et al., (2017). Thus, we test NH summer temperature CFRs employing a pseudo-proxy continental network that is the result of blending two networks: the PAGES2k Consortium multiproxy network, and the climate-tree-ring network of St. George (2014).

In the oceanic realm in the North Atlantic, additional marine proxy records based on mollusc shell bands (Pyrina et al., 2017) have been also used for climate reconstructions. These records, similarly to the dendroclimatological records, are based on annual growth bands, are annually resolved, and usually represent surface or subsurface water temperature. Compelling evidence has already been provided by earlier studies that Atlantic Ocean variability is an important driver of European summer climate variability (Jacobeit et al., 2003; Sutton and Hodson, 2005; Folland et al., 2009). Thus, we also employ an updated proxy network by combining the locations of marine proxies and tree ring proxies (Pyrina et al., 2017; PAGES 2k consortium, 2017; Luterbacher et al., 2016) to test the NAE summer seasonal temperature reconstructions.

95 The choice of climate model to run pseudo-experiments may plausibly have an impact on the estimation of method skills, since the spatial and temporal cross-correlations between climate variables may be model dependent. Thus, it is advisable to use 
several 'numerical laboratories' and employ several comprehensive Earth System Models (ESMs) to evaluate reconstructions methods. Besides, constructing PPEs based on different ESMs will highlight model-based impacts on the reconstructed magnitude and spatial patterns (Smerdon et al., 2011, Smerdon, 2012; Amrhein et al., 2020). Accordingly, in this study three different comprehensive Earth System Models are employed as 'surrogate climate database for setting up PPEs: the Community Climate System Model CCSM4, the Max-Planck-Institute climate model MPI-ESM-P and the Community Earth System Model CESM1-CAM5. Among the models providing climate simulations of the past millennium, these three models are the ones with the highest horizontal resolution.

\section{Data and Method}

\subsection{Data}

\subsubsection{Proxy data locations}

The pseudoproxies are constructed from the simulated grid-cell summer mean temperature sampled from three climate model simulations over the past millennium (see following subsections). In this context, 11 real proxy locations in the North AtlanticEuropean region (Pyrina et al., 2017; PAGES 2k consortium, 2017; Luterbacher et al., 2016) are selected for regional NAE

$110\left(60^{\circ} \mathrm{W}-30^{\circ} \mathrm{E}, 0-88^{\circ} \mathrm{N}\right)$ PPEs and 48 proxy locations across the Northern Hemisphere are chosen from the PAGES $2 \mathrm{k}$ network. The original North Hemisphere PAGES network was trimmed down by removing proxies that may show a combined temperature-moisture response and by selecting only one proxy among those deemed to be too closely located (and thus redundant from the climate model perspective). Specifically, the 48 dendrochronology locations are selected according to Figure 4 of St. George, S. (2014) which shows the correlation coefficient between the dendroclimatological proxy records and

115 summer season temperature. At most of the retained locations, the correlation between the dendroclimatological record and regional temperature is higher than 0.5 .

\subsubsection{Climate Models}

One of the climate models utilized in our study is the Max-Planck-Institute Earth System model MPI-ESM-P with a spatial horizontal resolution of about 1.9 degree in longitude and 1.9 degree in latitude. The simulation covers the period from 100

120 BC to 2000 CE. The model MPI-ESM-P consists of the spectral atmospheric model ECHAM6 (Stevens et al., 2013), the ocean model MPI-OM (Jungclaus et al., 2013), the land model JSBACH (Reick et al., 2013) and the bio-geophysical model HAMOCC (Ilyina et al., 2013). The setup of our simulations corresponds to the MPI-ESM-P LR setup in the CMIP5 simulations suite. However, since the present simulations does not belong to the CMIP5 project, the forcings used in this simulation and additional technical details are shown in the Appendix A.

125 The second climate model is the Community Earth System Model CESM1 Paleoclimate model CAM5 from the National Centre for Atmospheric Research (NCAR) (Otto-Bliesner et al., 2016) with a spatial resolution of 2.5 degree in longitude and 
1.9 degree in latitude (https://www.cesm.ucar.edu/projects/community-projects/LME/). The CESM-CAM5-LME simulation extends from $850 \mathrm{CE}$ to $2006 \mathrm{CE}$ using CMIP5 climate forcing reconstructions (Schmidt et al. 2011) and reconstructed forcing for the transient evolution of aerosols, solar irradiance, land use conditions, greenhouse gases, orbital parameters, and volcanic emissions. The atmosphere model employed in CESM1 is CAM5 (Hurrell et al., 2013), which is a significant advancement of CAM4 (Neale et al., 2013), whereas CCSM4 uses CAM4 as its atmospheric component. The CESM1-CAM5 uses the same ocean, land and sea ice models as CCSM4 (Hurrell et al., 2013) does. We use one simulation form the Last Millennium Ensemble (LME).

The third climate model is the Community Climate System Model CCSM4 model (Gent et al., 2011), also from NCAR, which uses the land (CLM4/ Lawrence et al., 2012), the ocean model(POP2/Smith et al., 2010)the atmosphere (CAM4/Neale et al., 2013) and the sea ice model (CICE4/Hunke et al., 2008) components. CCSM4 is modelled with a spatial resolution of 1.25 degree in longitude and 0.9 degree in latitude. Here, we use the simulations labelled past 1000 and historical and labelled r1ilp1 from the CMIP5 data set. The past 1000 simulation spans the period from $850 \mathrm{CE}$ to $1849 \mathrm{CE}$. The historical simulations covers the period $1850 \mathrm{CE}$ to $2005 \mathrm{CE}$. We concatenate both simulations together for this study. The boundary conditions and forcings follow the PMIP3 (Paleoclimate Modelling Intercomparison Project Phase III) protocols (Schmidt et al. 2011). The Lean et al. (2005) reconstruction of the total solar irradiance (TSI) combined with the Vieira et al. (2011) is employed to describe the TSI. The volcanic forcing is prescribed by using the ice-core-based index of Gao et al. (2008). The Pongratz et al. (2008) reconstruction of land use change merged with that of Hurtt et al. (2009) is used to describe seamlessly land use changes.

\subsection{Methods}

\subsubsection{Construction of pseudo-proxies}

To test the statistical reconstruction methods in the virtual laboratories of climate model simulations, we need records that mimic the statistical properties of real proxy records. The most important properties are their correlation to the local temperature and their location in a proxy network. A third important characteristic is the network size and temporal coverage. The usual method to produce pseudo-proxy records in climate simulations is to sample the simulated temperature at the gridcell co-located with the real proxy record and contaminate the simulated temperature with added statistical noise, so that the correlations between the original temperature and the contaminated temperature resembles the typical temperature-proxy correlations. The real correlation is of the order of 0.5 or above for good proxy records. This parameter can be modulated in the pseudo-proxy record by the amount of noise added to the simulated temperature, and different proxy networks will help us to reveal how and to what extent degradations and effects would be expressed amongst methods and amongst climate models. Ideal pseudo-proxies contain only the temperature signal subsampled from the climate model. We then perturb the ideal pseudo-proxies with Gaussian white noise. Gaussian white noise with signal-to-noise ratio (SNR) values of 0.25, 0.5 and 1 is in general widely employed for contaminating the ideal pseudoproxy dataset (Smerdon, 2012; Wang et al., 2014). The noise level can be defined using various criteria including SNR, variance of pure white noise (NVAR), and percent noise by variance 
(PNV) and so on (Smerdon, 2012). We employ here the PNV to define the noise level convention; The PNV expresses the ratio between the added noise variance and the total variance of resulting the pseudo-proxy time series.

Although individual real proxies contain different amounts of noise (non-climatic variability), we assume here a uniform level of noise throughout the whole pseudo-proxy network. In addition, real proxy records contain temporal gaps, and not all records span the same period. For the sake of simplicity, we assume in our pseudo-proxies network that the data have no temporal gaps and all records cover the whole period of the simulations

165 The dataset employed herein for constructing PPEs database is split into a calibration period that spans 850-1425AD, and a validation period that spans 1426-2000 AD. All the validation statistics of the CFR results are derived against the reconstruction period of 1426-2000 AD. Note that here we just split the entire temporal interval into two equal parts, while, in reality, the instrumental period only covers the most recent 150 years. It means that in reality, only the most recent 150 years can be used for training of the statistical models. The reconstruction skill derived in this study will, all other factors being equal, be larger than the real reconstruction skill, since the data available for calibration are less and the calibration period contains different climate regimes, e.g. the strong anthropogenic warming signal which is not present in the preindustrial period. On the other hand, we exclude the period of strong anthropogenic warming from the calibration data, so that we present to the methods the clear challenge of reconstructing the temperature beyond the range of variations seen in the calibration period, expecting that the possible deficiencies of the reconstruction methods become more apparent with this set-up.

\subsubsection{Principal component regression}

Principal component analysis is employed to construct a few new variables that are a linear combination of the components of the original climate field, and that ideally describe a large part of the total variability. The linear combinations that define the new variables are the eigenvectors of the cross-covariance matrix of the field. Associated to each variable (eigenvector), a principal component time series (scores) describes its temporal variation. In the PCR, the predictands are those scores identified by PCA of the climate field (Hotelling, 1957; Luterbacher et al., 2004; Pyrina et al., 2017). This results in a reduction of dimensionality without losing too much information, and reduces the risk of over-fitting. In the present study, the retained PCs capture at least $90 \%$ of the cumulative temporal variance of climate field. After selecting the empirical orthogonal functionsEOFs and principal components-PCs based on the calibration dataset and establishing the desired linear regression relationships between the PCs and the proxy dataset (predictors), the PCs in the validation period are reconstructed using the estimated regression coefficients. The full climate field is then reconstructed by the linear combination of the reconstructed PCs and their corresponding EOFs. A given climate field $\boldsymbol{x}_{\mathrm{t}}$, at time step $t$ can be decomposed as follows:

$\boldsymbol{x}_{m, t}=\sum_{n=1}^{k} P C_{n, t} \boldsymbol{E O F}_{m, n}$

where $m$ is the grid index of the field, $t$ is the time index, and $k$ denotes the total numbers of retained PCs.

The linear relationship between proxies and targeted climate field is established by the regression equation: 
where the index $m$ runs over the proxies, $j$ denotes the total numbers of proxies, $\omega$ is the linear function coefficient, and $\varepsilon$ denotes a residual term. The $\omega$ parameters are estimated by Ordinary Least Squares. Here, it is assumed that climate sensitive proxies are linearly related with the climate PCs. Based on Eq. (3) using the PCR method, the PCs during the validation interval will be reconstructed assuming that the linear coefficients derived in Eq. (3) are constant in time:

The final reconstructed field $\hat{x}$ will be derived by the linear combination of the reconstructed $\widehat{P C}$ with the EOFs derived from the calibration dataset, thereby assuming that the EOF patterns remain constant in time (Gómez-Navarro et al., 2017; Pyrina et al., 2017).

\subsubsection{Canonical correlation analysis}

Canonical Correlation Analysis CCA is also an eigenvector method. Similarly to PCA, CCA decomposes the variance of the fields as a linear combination of spatial patterns and their corresponding amplitude time series. In contrast to PCA, where the target is to maximize the explained variance with a few new variables, CCA constructs pairs of predictor-predictand variables that maximize the temporal correlation of the corresponding amplitude time series. The pairs of variables are identified by solving an eigenvalue problem that requires the calculation of the inverse of the covariance matrices of each field. These matrices can be pseudo-degenerate (one eigenvalue much smaller than the largest eigenvalue) and therefore the calculation of their inverse is, without regularization, numerically unstable. This regularization can be introduced by first projecting the original fields onto their leading EOFs (Widmann, 2005; Pyrina et al., 2017). This also reduces the number of degrees of freedom - thus hindering overfitting - and eliminate potential noise variance. After the dimensional transformation, a small number of pairs of patterns with high temporal correlation will be retained. In the present study, the number of retained PCs capture at least $90 \%$ cumulative variance of predictand climate field. Then these retained PC time series will be used as input variables of CCA to calculate the canonical correlation patterns (CCPs) and canonical coefficients (CCs) time series for both proxy and temperature field. The reconstructed climate field can be calculated by a linear combination of the CCPs with CCs for each time step $t$ :

$\boldsymbol{x}_{m, t}=\sum_{n=1}^{l} C C_{n, t}^{\text {field }} \boldsymbol{C} \boldsymbol{C} \boldsymbol{P}_{m, n}^{\text {field }}$

$\boldsymbol{P r o x y}_{m, t}=\sum_{n=1}^{l} C C_{n, t}^{\text {proxy }} \boldsymbol{C C P}_{m, n}^{\text {proxy }}$

Proxy denotes the reconstructed proxy field, and $l$ is the number of CCA pairs. The correlation between each pair CC (proxy, field) are the canonical correlations, which are the square root of the CCA-eigenvalues. Therefore, once each $C C^{\text {proxy }}(t)$ is calculated from the proxy data through the validation period, the corresponding $C C^{f i e l d}(t)$ can be easily estimated as proportional to $C C^{\text {proxy }}(t)$, since the correlation between the different $C C_{n}{ }^{\text {proxy }}(t)$ is zero. The final reconstruction of target climate field will be derived by linear combination of $C C P^{\text {field }}(t)$ and $C C^{\text {field }}(t)$, assuming again that the dominant canonical correlation patterns of climate variability are stationary in time. 


\subsubsection{Bidirectional Long Short-term memory neural network}

As a non-linear machine learning method, we test here a Bidirectional Long short-term memory neural network (Bi-LSTM). The LSTM networks, in contrast to the more traditional neural networks, also capture the information of the serial co-variability present in the data, and therefore are suitable to tackle data with a temporal structure. They are usually applied to the analysis of speech and of time series. The rationale of using these type of networks for climate reconstructions is the aforementioned underestimation of the past climate variations by most linear methods ('regression to the mean'). In principle, a LSTM network could exploit the temporal autocorrelation present in the time series to ameliorate this underestimation and perhaps also provide more realistic spectral properties of the reconstructed time series.

230 The structure of LSTM network is more complicated than the structure of a traditional neural network. The LSTM estimates a hidden variable $h(t)$ that encapsulates the state of the system at time $t$. The computation of the new system state at time $t+1$, $h(t+1)$, depends on the value of the predictors at $t+1$ but also on the value of the hidden state at time $t, h(t)$. The training of the LSTM can be accomplished sequentially by assimilating the information present in the training data from time steps in the past of the present time step. In some loose sense, a LSTM network would be the machine-learning equivalent of a linear autoregressive process.

The training of the network is accomplished by feeding it with sequential data iteratively, forwards towards the future and backwards towards the past. Both forward and backward assimilations are processed by two separated LSTM neural layers, which are connected to the same output layer. Figure 1 illustrates the bidirectional structure of the Bi-LSTM network. Given a set of predictor-predictand variables $\left(\boldsymbol{X}_{\boldsymbol{t}}, \boldsymbol{Y}_{\mathrm{t}}\right)$, our goal is to train a nonlinear function:

The structure of this complex non-linear function $F$ is defined as follows:

$f_{t}=\sigma\left(W_{f}\left[h_{t-1}, x_{t}\right]+B_{f}\right)$

$i_{t}=\sigma\left(W_{i}\left[h_{t-1}, x_{t}\right]+B_{i}\right)$

$A_{t}=\tanh \left(W_{A}\left[h_{t-1}, x_{t}\right]+B_{A}\right)$

$C_{t}=f_{t} C_{t-1}+i_{t} A_{t}$

$o_{t}=\sigma\left(W_{o}\left[h_{t-1}, x_{t}\right]+B_{o}\right)$

$h_{t}=o_{t} \tanh \left(C_{t}\right)$

where $W_{f}, W_{i}, W_{A}$ and $W_{o}$ represent several weight matrices and $B_{f}, B_{i} B_{A}$ and $B_{o}$ represent different bias matrices. $\sigma$ is the gate activation function, here we utilize the Rectified Linear Unit function-ReLU (Ramachandran et al., 2017) .

250 At time step $t-1$, the hidden state of LSTM cell's hidden layer is preserved as $h_{t-1}$, and this vector is combined with the vector of current input variables $\boldsymbol{X}_{t}$ to obtain the state of the forget gate, $f_{t}$ (equation 7), the input gate $i_{t}$ (equation 8) and the state of 
memory cell $A_{t}$ (equation 9). This memory cell state $A_{t}$ is linearly combined with the previous state of the cell output $C_{t-1}$ to update the value of its state. The weights of this linear combinations are the states of the forget gate $f_{t}$ and of the input gate $i_{t}$ (equation 10). The state of the output gate $o_{t}$ is calculated from the previous hidden state and the current input variables (equation 11). This output is used to compute the updated hidden state $h_{t}$ using the state of the cell output $C_{t}$ (equation 12) (Huang et al., 2020; Chattopadhyay et al., 2020).

In the present application to climate reconstructions, we have a set of input pseudoproxy data $\boldsymbol{X}_{t}^{n}=\left[x_{\mathrm{t}-\mathrm{i}}, \ldots, x_{\mathrm{t}-1}\right]$ and an output target temperature time series $\boldsymbol{Y}_{t}^{m}=\left[y_{\mathrm{t}-\mathrm{i}}, \ldots, y_{\mathrm{t}-1}\right]$. The forward LSTM hidden state sequence $\overrightarrow{\boldsymbol{h}_{t}}$ (note the arrow direction) is calculated employing inputs information in a positive direction from time $t$ - 1 to time $t$ - $n$ iteratively, and for backward LSTM cell, the hidden state sequence $\overleftarrow{\boldsymbol{h}_{t}}$ is computed using the input within a reverse direction from time $t$ - $n$ to time $t$ - 1 iteratively. The final outputs from the forward and backward LSTM cells are calculated utilizing the calculation equation (Cui et al., 2018, Jahangir et al., 2020):

$\widetilde{\boldsymbol{Y}}_{t}=\operatorname{concat}\left(\widetilde{\boldsymbol{h}_{t}}, \overleftarrow{\boldsymbol{h}_{t}}\right)$

where concat is the function used to concatenate the two output sequences $\overrightarrow{\boldsymbol{h}}$ and $\overleftarrow{\boldsymbol{h}}$ (Cui et al., 2018, Jahangir et al., 2020).

265 During training process, the calibration dataset are fed into LSTM cell, and it will map the potential latent relationships (both linear and nonlinear) between input and output variables by updating its weight and threshold matrices. The object function for Bi-LSTM to be minimized during training is the Huber loss that expresses the mismatch between the reconstructed climate field and the 'real' climate field from model simulations. Object loss is optimized by gradient descent (Goodfellow et al., 2016). Huber loss has a key advantage of being less sensitive to outlier values:

$L_{\delta}(\boldsymbol{Y}, f(\boldsymbol{X}))=\left\{\begin{array}{c}\frac{1}{2}(\boldsymbol{Y}-f(\boldsymbol{X}))^{2} \\ \delta|\boldsymbol{Y}-f(\boldsymbol{X})|-\frac{1}{2} \delta^{2}\end{array}\right.$

where $f$ denotes the neural network and the brackets denote the Euclidean norm. The Huber loss function changes from a quadratic to linear when $\delta$ (a positive real number) varies from small to big (Meyer, 2020). Huber loss will approach L2 loss when $\delta$ tends to be 0 , and approach L1 when $\delta$ tends to be positive infinity, here we test its value and finally set $\delta 1.35$. L2 is the square root of the sum of squared deviations and L1 is the sum of absolute deviations.

275 The main mechanism of LSTM is that the LSTM block manages to develop a regulated information flow by controlling which proportion of data information should be 'remembered' or should be 'forgotten. By controlling the regulation of the information flow, LSTM will manage to learn and preserve temporal characteristics and dependencies of the specific time series.

In this particular application, we have used two hidden Bi-LSTM layers with 700 neurons each (some additional tests on selecting the number of neurons in the hidden layer are shown in Appendix B). The optimization function is Adam with the learning rate of 0.0001 (Kingma and $\mathrm{Ba}, 2014$ ). After training the model, the optimal network hyper-parameters, including 
weights and threshold matrices, are estimated. The validation pseudoproxy dataset is then used to reconstruct the target temperature field in the validation directly.

\section{Results}

285 We evaluate the reconstruction skill of the different methods based on the Pearson correlation coefficient (cc) between each target series and the corresponding reconstructed series, and their Standard deviation ratio (SD ratio, SD ratio = $\mathrm{SD}_{\text {reconstruction }} / \mathrm{SD}_{\text {model }}$ ). All the evaluation metrics are calculated in the validation period from 1426-2000 AD. High values of derived cc indicate better temporal covariance between target and reconstructed results, a high SD ratio denotes that more variance is preserved in the reconstructions. Usually, the reconstructed variance is smaller than the target variance and thus their ratio is usually smaller than unity.

\subsection{North Atlantic-Europe CFRs}

Fig. 2 illustrates the CFR results for the North Atlantic-European region employing the 11 ideal-noise-free pseudoproxies based on the three CFR methodologies and the three climate model simulations. When comparing the reconstruction skills across these three CFR methods derived with the same climate model (for example, MPI, CAM5 or CCSM correspondingly), all the spatial cc patterns exhibit similarities. This indicates that all three CFR methods show generally reasonable spatial reconstruction skills (mean cc's over the entire NAE are bigger than 0.45). In addition, cc maps show higher values over regions where more pseudoproxies are located. This confirms the well-documented tendency amongst different multivariate linear based regression methods to better reconstruction skill in the sub-regions with denser pseudoproxy sampling than in regions with sparser networks (Steiger et al., 2014; Evans et al., 2014; Wang et al., 2014). The cc pattern of the nonlinear method Bi-LSTM is very similar to that of the linear methods, even though the structure of the statistical models is very different. This shows that the nonlinear method employed herein has the similar tendency as linear models to obtain better reconstruction skill over denser proxy sampling regions.

The picture that emerges from the SD ratio is also very similar for the three methods (Fig. 2). In the regions with a high pseudo proxy density, the SD ratio is high, but outside of the densely sampled areas, all three CFR methods experience a similar degree

305 of variance loss.

More realistic pseudo-proxies are those containing 80\% Gaussian white noise contamination. This amount of noise is constructed and added to the ideal temperature signal of the 11 pseudoproxies subsampled from MPI, CAM5 and CCSM models. The corresponding spatial $\mathrm{cc}$ and SD ratio patterns are displayed in Fig. 3. Compared to reconstructions with ideal pseudo proxies (Fig. 2), a strong degradation of reconstruction skill amongst all CFR methods occurs over the entire NAE.

310 The degradation is especially profound in the regions where denser pseudoproxies exist (the mean cc is reduced from 0.8 in the ideal PPEs to approximately 0.3 in the noisy PPEs). Weak reconstruction skill (approximately mean cc is 0.3 , and SD ratio 
https://doi.org/10.5194/cp-2022-5

Preprint. Discussion started: 31 January 2022

(C) Author(s) 2022. CC BY 4.0 License.

\section{(c) (i)}

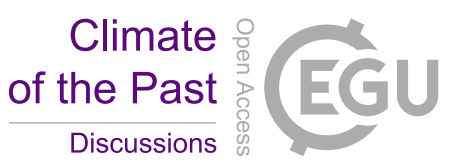

is 0.6) exists over regions where proxies are available, together with a small surrounding region. These noise contamination results shown in Fig. 3 demonstrate again that the nonlinear method exhibit CFR similarities to the linear methods.

\subsection{Northern Hemisphere CFRs}

$315 \mathrm{NH}$ summer temperature anomalies reconstructions based on PPEs using three CFR methodologies and the three climate models are displayed in Fig. 4-5.

Table 1. Skill reconstruction statistics for the North Hemisphere mean temperature in the verification period for ideal PPEs. The table shows the result for three CFR methods (PCR, CCA and Bi-LSTM) and three climate models (MPI, CAM and CCSM). The numbers in parenthesis indicate the skill statistics of $80 \%$ noise contaminated PPEs.

\begin{tabular}{ccccccc}
\hline \multirow{2}{*}{ Method } & \multicolumn{3}{c}{ SD Ratio } & \multicolumn{3}{c}{ cc } \\
\cline { 2 - 7 } PCR & MPI & CAM & CCSM & MPI & CAM & CCSM \\
CCA & $0.642(0.427)$ & $0.713(0.486)$ & $0.732(0.483)$ & $0.594(0.333)$ & $0.636(0.372)$ & $0.709(0.467)$ \\
Bi-LSTM & $0.557(0.388)$ & $0.612(0.457)$ & $0.604(0.443)$ & $0.565(0.333)$ & $0.587(0.370)$ & $0.648(0.457)$ \\
& $0.604(0.375)$ & $0.729(0.425)$ & $0.719(0.412)$ & $0.556(0.319)$ & $0.611(0.371)$ & $0.660(0.456)$ \\
\hline
\end{tabular}

The spatial cc maps and SD ratio distributions for the ideal PPEs in NH are shown in Fig. 4. Again, all three CFR methodologies yield relatively similar spatial patterns of skill for each of the climate models employed here. Skilful reconstructions are again achieved over regions with a denser pseudoproxy network (over North American and Eurasia regions). In addition, relatively large SD ratio and high cc values also occur in tropical regions. These regions barely contain any pseudoproxies, indicating that the climate teleconnections between tropics and mid-latitude regions could be responsible for the reconstruction skill in tropical regions.

All derived CFRs suffer from variance losses as shown in Fig. 4 and in Table 1. The spatial distributions of the SD ratio vary between climate models and CFR methodologies. They also are spatially heterogeneous. The most important factor affecting the ratio of standard deviations seem to be again the chosen climate model. For instance, comparing the spatial patterns of SD ratio in Fig. 4 across the three models, more variance in the high-density pseudoproxy sampling and the tropical regions are preserved in the high-resolution CCSM model across all CFR methods, whereas, CAM5 and MPI model suffer from relatively more variance loss over these areas. In addition, the highest mean cc is obtained by all three methods in the CCSM simulation (See Table 1).

The Bi-LSTM and PCR methods exhibit relatively consistent patterns with similar SD ratios. The CCA methodology seems to suffer more strongly from variance losses (see Table 1) over the entire NH compared to PCR and Bi-LSTM.

The NH results obtained from the $80 \%$ noise contaminated PPEs are displayed in Fig. 5. The performance deterioration is expected and again significant. The reduction of reconstruction skill also occurs over regions where dense pseudoproxies are located. The nonlinear method Bi-LSTM seems to suffer more strongly from variance losses over the NH (see Table 1), 
https://doi.org/10.5194/cp-2022-5

Preprint. Discussion started: 31 January 2022

(C) Author(s) 2022. CC BY 4.0 License.

especially over the North Atlantic region (Fig. 5) across all three models. This might indicate that the nonlinear method could

be more sensitive to the noise contamination than the other methods.

In addition, a relatively better performance is derived, also in the case of noisy pseudoproxies, from the CCSM using all CFR methods (see the spatial cc patterns shown in Fig. 5 and mean cc values in Table 1).

Considering the general methodological skill, as indicated by the derived cc and SD ratio values in Table 1, the Bi-LSTM method presents relatively worse performance with lower mean cc and SD ratio. The methods PCR and Bi-LSTM generally outperform the CCA methodology with higher mean cc with ideal PPEs. Overall, PCR generally outperforms CCA and BiLSTM with highest mean cc and SD ratio values across all PPEs in all three model simulations.

\subsection{Northern Hemisphere and AMV indices}

The evolution of the decadal NH mean temperature anomalies reconstructed by the three CFR methodologies and using perfect pseudoproxies from three models is illustrated in Fig. 6 on the left panel. All indices have been smoothed using a Butterworth low-pass filter to remove temporal fluctuations shorter than 10 years. The reconstruction performance varies amongst different CFR methodologies.

Table 2. RMSE (K) and cc during the verification interval for decadal NH mean temperature derived from ideal PPEs. The numbers in parenthesis indicate the RMSE $(\mathrm{K})$ and cc of $80 \%$ noise contaminated PPEs.

\begin{tabular}{ccccccc}
\hline \multirow{2}{*}{ Method } & \multicolumn{5}{c}{ RMSE } & \multicolumn{3}{c}{ cc } \\
\cline { 2 - 7 } PCR & MPI & CAM & CCSM & MPI & CAM & CCSM \\
CCA & $0.102(0.217)$ & $0.066(0.119)$ & $0.062(0.186)$ & $0.974(0.872)$ & $0.913(0.691)$ & $0.991(0.939)$ \\
Bi-LSTM & $0.124(0.226)$ & $0.075(0.120)$ & $0.108(0.200)$ & $0.967(0.867)$ & $0.897(0.669)$ & $0.976(0.931)$ \\
& $0.123(0.239)$ & $0.067(0.116)$ & $0.109(0.222)$ & $0.941(0.797)$ & $0.908(0.698)$ & $0.971(0.877)$ \\
\hline
\end{tabular}

355 Table 3. The same as Table 2, but for decadal AMV index

\begin{tabular}{ccccccc}
\hline \multirow{2}{*}{ Method } & \multicolumn{5}{c}{ RMSE } & \multicolumn{3}{c}{ cc } \\
\cline { 2 - 7 } PCR & MPI & CAM & CCSM & MPI & CAM & CCSM \\
CCA & $0.106(0.209)$ & $0.073(0.114)$ & $0.071(0.175)$ & $0.959(0.847)$ & $0.883(0.691)$ & $0.979(0.933)$ \\
Bi-LSTM & $0.129(0.217)$ & $0.078(0.118)$ & $0.117(0.185)$ & $0.946(0.844)$ & $0.875(0.665)$ & $0.959(0.929)$ \\
& $0.123(0.231)$ & $0.081(0.115)$ & $0.098(0.204)$ & $0.927(0.769)$ & $0.843(0.682)$ & $0.969(0.883)$ \\
\hline
\end{tabular}

The temporal evolution of the AMV index (the right panel in Fig. 6) differs among the simulations, reflecting the different forcings used in each simulation and the model specific contribution of internal variability to the index variations (Wagner and Zorita, 2005; Schmidt et al., 2011). Considering the methodological performance, all three methods generally achieve good 
https://doi.org/10.5194/cp-2022-5

Preprint. Discussion started: 31 January 2022

(C) Author(s) 2022. CC BY 4.0 License.

AMV index reconstructions when using perfect pseudo-proxies, as shown in each subfigures of Fig. 6, and also refer the RMSE in Table 3.

The NH and AMV indices derived from more realistic 80\% noise contaminated CFRs are shown in Fig. 7. The larger noise contamination results in substantial skill deterioration (RMSD and cc displayed within brackets in Table 2 and 3). All three methods fail to capture the warming trend over the most recent century, and the magnitude of strong cooling events is strongly underestimated.

\subsection{Probability distributions of reconstructed variables}

Table 4. Kolmogorov-Smirnov test statistic and p-value for quantifying the histogram distributions between model and reconstructed NH decadal means. Low values of the KS statistic indicate larger similarity between the two distributions. The numbers in parenthesis indicate the KS statistic and p-value of $80 \%$ noise contaminated PPEs.

\begin{tabular}{ccccccc}
\hline \multirow{2}{*}{ Method } & \multicolumn{3}{c}{ KS statistic } & \multicolumn{3}{c}{ p-value } \\
\cline { 2 - 7 } & MPI & CAM & CCSM & MPI & CAM & CCSM \\
PCR & $0.092(0.213)$ & $0.111(0.246)$ & $0.053(0.128)$ & $1 \mathrm{e}-2(6 \mathrm{e}-12)$ & $1 \mathrm{e}-3(8 \mathrm{e}-16)$ & $3 \mathrm{e}-1(1 \mathrm{e}-3)$ \\
CCA & $0.111(0.246)$ & $0.142(0.252)$ & $0.072(0.149)$ & $2 \mathrm{e}-3(8 \mathrm{e}-16)$ & $1 \mathrm{e}-5(1 \mathrm{e}-16)$ & $9 \mathrm{e}-2(5 \mathrm{e}-6)$ \\
Bi-LSTM & $0.135(0.269)$ & $0.126(0.253)$ & $0.125(0.229)$ & $4 \mathrm{e}-5(8 \mathrm{e}-19)$ & $1 \mathrm{e}-4(1 \mathrm{e}-16)$ & $2 \mathrm{e}-3(1 \mathrm{e}-13)$ \\
\hline
\end{tabular}

Table 5. The same as Table 4, but for AMV index.

\begin{tabular}{ccccccc}
\hline \multirow{2}{*}{ Method } & \multicolumn{3}{c}{ KS statistic } & \multicolumn{3}{c}{ p-value } \\
\cline { 2 - 7 } & MPI & CAM & CCSM & MPI & CAM & CCSM \\
PCR & $0.116(0.224)$ & $0.123(0.198)$ & $0.046(0.126)$ & $8 \mathrm{e}-4(4 \mathrm{e}-13)$ & $3 \mathrm{e}-4(2-\mathrm{e} 10)$ & $5 \mathrm{e}-1(2 \mathrm{e}-4)$ \\
CCA & $0.116(0.248)$ & $0.118(0.215)$ & $0.076(0.145)$ & $8 \mathrm{e}-4(5 \mathrm{e}-16)$ & $6 \mathrm{e}-4(4 \mathrm{e}-12)$ & $6 \mathrm{e}-2(9 \mathrm{e}-6)$ \\
Bi-LSTM & $0.151(0.262)$ & $0.125(0.212)$ & $0.093(0.197)$ & $3 \mathrm{e}-6(7 \mathrm{e}-18)$ & $2 \mathrm{e}-4(12 \mathrm{e}-12)$ & $1 \mathrm{e}-2(3 \mathrm{e}-10)$ \\
\hline
\end{tabular}

Even though the three reconstructions methods tend to underestimate the overall variability when using noisy pseudoproxies, an interesting question is their skill in reproducing the probability distributions of the climate indices. In particular, a relevant question is whether the methods are able to capture extreme phases of those indices.

Fig. 8 and 9 display the histogram for decadal NH mean and AMV index, respectively. Each subfigure represents the histograms of reconstructed temperature indices across the three methods, compared with the histograms of the target temperature index.

For perfect pseudoproxies, the PCR reconstruction seems to capture the overall target distribution best. It captures the lower tail better than CCA and the upper tail better than CCA and Bi-LSTM. The differences between the methods become smaller for the reconstructions with noisy pseudo proxies, with the PCR still being better than the other two methods (subfigures for 
https://doi.org/10.5194/cp-2022-5

Preprint. Discussion started: 31 January 2022

(c) Author(s) 2022. CC BY 4.0 License.

(c) (i)

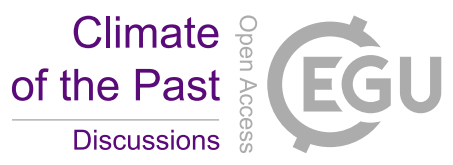

the contaminated PPEs in Fig. 8 and 9). The Bi-LSTM captures the lower tails of distribution much better than the upper tails, both for the NH mean and the AMV index.

We also quantify the distribution similarity between reconstructed and target distributions for both $\mathrm{NH}$ and AMV indices using the two-sample Kolmogorov-Smirnov test as a metric (Hodges, 1958) (see Table 4-5). The smallest KS statistic is achieved by the PCR method (see Table 4-5), confirming the impression that the PCR outperforms the other two methods in both the ideal and noise contaminated PPEs.

\section{Discussions}

\subsection{Nonlinear method performance}

390 As the Bi-LSTM method is the most complex of the three tested in this study, it is reasonable to expect a better reconstruction skill. However, this is not the case in our pseudoproxy experiments. For the spatially resolved NAE and NH fields, the nonlinear Bi-LSTM method achieves a similar skill as the linear PCR and CCA methods. Whereas the spatial SD ratio and cc present CFR are generally very similar, the PCR is generally the best method among the three methods, with the nonlinear BiLSTM as second best method (see mean skill statistics in Table 1).

395 For the area-mean indices, all three methods exhibit again similar skill. The Bi-LSTM is able to capture periods of extreme cooling better than the other two methods but strongly underestimates the recent warming trend. The inability to capture the warming trend indicates that the Bi-LSTM is not good at extrapolating to temperature ranges beyond the training set. Nonetheless, even though the traditional PCR seems to display the overall best performance, the fact that the Bi-LSTM is able to capture some extremes better is encouraging. This indicates that there may not be one sole reconstruction method which captures the mean and the extremes equally well. In addition, Guillot et al. (2015) constructed different CFR methods for exploring the disagreement between climate field reconstructions and area mean index reconstructions. They demonstrated that the skill for the regionally averaged time series is a relatively poor indicator of the spatial performance.

Nonlinear methods are usually capable of mapping complex systems with high nonlinearity. Here, we employ one nonlinear neural network method Bi-LSTM to test its performance on CFR reconstructions. Compared with linear methods PCR and

405 CCA, neural network model did not show clear advantages, except for capturing extremes in the lower tail of the distribution. It is possible that the performance of the Bi-LSTM could be further improved by optimizing the architecture and parameters of the network, including the type of object function, type of neural activation function, network optimization function, number of hidden layers, the model-learning rate etc. At this point, it would be quite natural to consider whether the selection/settings of these hyper-parameters in our study is optimal, and also to what extent the reconstruction skill is sensitive to changes in the

410 hyper-parameters. Nadiga (2020) pointed out that the skill of some machine learning-methods are strongly dependent on these hyper-parameters. Machine learning methods include an extensive range of complexity, and therefore it remains an open issue as to which ML techniques are most or relatively suitable for paleoclimate. It is not clear how the structure of the machinelearning methods can be systematically optimized. At the moment, there is still a considerably amount of 'trial and error' in 
https://doi.org/10.5194/cp-2022-5

Preprint. Discussion started: 31 January 2022

(c) Author(s) 2022. CC BY 4.0 License.

(c) (i)

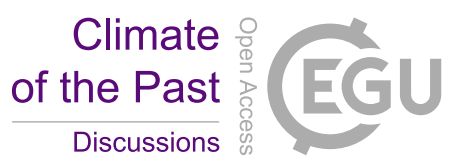

the design and connection of the neural layers. Here, we have tested the Bi-LSTM network with a relatively simple architecture

of two separated hidden layers, and evaluated its performances on CFR experiments, which could be a preliminary try. It is, however possible that more complex models or architectures (Kadow et al., 2020) might achieve better or comparable skill in CFR experiments. Thus, more different theory-based and architecture-based machine learning methods might be worth exploring in future studies.

\subsection{Model and pseudoproxy experiment dependency}

420 The evaluation of the reconstruction skill seems to depend as much on the reconstruction method as on the underlying climate model simulation from which the pseudoproxies were generated. The differences in skill for the same method with different climate model data is of the same order as the differences in skill for the different methods with the same climate model data. The performance of the method does not seem to depend on the domain of the reconstruction. The reconstructions behave very similar for both the NAE and the NH test cases.

425 Considering the effects of noise contamination on the methodological performance, all three methods display similar skills in the ideal PPEs, but all methods suffer from variance underestimation and lower correlation coefficients in the more realistic PPEs (80\% noise contaminated PPEs). The nonlinear Bi-LSTM is more strongly impacted by the noise contamination (Table 1).

From the perspective of the spatial coverage of the proxy network, the spatial cc and SD ratio patterns reveal reconstruction

430 skill over the entire NH regions, although this skill is weaker in areas more poorly sampled by the pseudo-proxy network. Interestingly, the tropical regions do show some reconstruction skill, although almost not pseudo-proxies are located in the Tropics. In addition, the reconstruction methods achieve better reconstruction skill when evaluated with the climate model with highest (most realistic) spatial resolution. This result indicates the climate teleconnections between tropics and midlatitude regions could lead to some indirect skill, and that in the real world this indirect reconstruction skill could be larger than that obtained in our pseudo-proxy experiments. However, the proxy networks and noise scenarios constructed in the context are certainly not able to mimic/simulate the full range of characteristics completely for climatic proxies in the real world.

\section{Conclusions}

A nonlinear Bi-LSTM neural network method to reconstruct North Atlantic-Europe and Northern Hemisphere temperature 440 fields was tested with climate surrogate data generated by simulations with three different climate models. Compared to the more classical methods of linear Principal Components Regression and Canonical Correlation Analysis, the NAE and NH summer temperature field could be reasonably reconstructed using both linear and nonlinear methodologies. All three methods show skill similarities in both NAE and NH PPEs. 
https://doi.org/10.5194/cp-2022-5

Preprint. Discussion started: 31 January 2022

(c) Author(s) 2022. CC BY 4.0 License.

(c) (i)

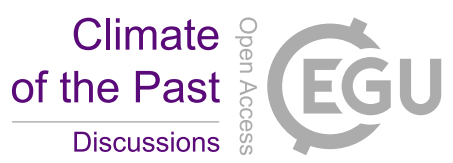

In general, all three methods display similar skills when using ideal (noise-free) pseudoproxies, while in the more realistic

PPEs (80\% noise contaminated PPEs), the Nonlinear Bi-LSTM seems to suffer more strongly from variance losses. This might indicate that the nonlinear method would be more sensitive to the noise contamination than the other methods.

The pseudoproxy networks used in this study were mostly located in the extratropical regions with only three proxies in the tropical area. All CFR methodologies produce generally good reconstructions in regions where dense pseudoproxy networks are available. Moreover, teleconnections are explored by these CFR methodologies, leading to some weak spatial reconstruction skills outside of the proxy-sampled regions, for instance the tropical region.

The classical linear-based PCR method generally outperforms the Bi-LSTM and CCA method in both spatial and index reconstructions. However, the Bi-LSTM seems to be able to capture extreme cooling events better than the other two methods, while failing to capture the warm tails of the temperature. In particular, this is reflected in the inability of the non-linear method to replicate the $20^{\text {th }}$ century warming trend.

Here, we could draw a general conclusion that nonlinear artificial neural network method Bi-LSTM employed herein is not superior for CFR reconstructions, at least in our PPEs. In general, Bi-LSTM show worse skill in spatial and temporal CFRs than PCR and CCA, also in capturing extremes. Yet, it is essential to employ a large range of nonlinear CFR methods to evaluate different model structures, and further test their performance on CFRs. For example, additional nonlinear-based regression methodologies, convolutional neural network and one of the widely implemented Reservoir Computing methodsEcho State Network, could be techniques with powerful non-linear regression capability for paleoclimate field reconstructions.

\section{Appendix A}

The simulation with the model MPI-ESM-P is not part of the standard CMIP5 simulation suite. In the following, we include additional technical details on this simulation. The MPI simulation was started from the year of $100 \mathrm{BC}$ with restart files from a 500-year spin-down simulation experiments forced with constant external conditions representing the year 100 of BC. After $100 \mathrm{BC}$, variation in volcanic, solar, orbital, and GHG concentrations are implemented. Land usage was held constant until $850 \mathrm{AD}$ with conditions representing those for year $850 \mathrm{AD}$. The variation of orbital parameters are calculated after the PMIP3protocol (Schmidt et al. 2011). The solar activity has been rebuilt on the basis of the reconstruction of Vieira et al. 2011 employing the algorithm and scaling outlined in Schmidt et al. 2011 which corresponds to a difference in short-wave top of the atmosphere insolation of $1.25 \mathrm{Wm}-2(\sim 0.1 \%)$ between the 2 nd half of the 20th century $(1950-2000)$ and the Maunder Minimum (1645 - 1715). Variations in greenhouse gas concentrations related to CO2, N2O and CH4 are after the reconstruction of the PMIP3 protocol - The concentrations were held constant to the values of year $1 \mathrm{AD}$ between $100 \mathrm{BC}$ and $1 \mathrm{AD}$ because the law Dome records does not extend beyond year $1 \mathrm{AD}$. After $1850 \mathrm{AD}$ also a reconstructed aerosol loading after Stine et al. 2018 were employed to account for transient anthropogenic aerosol emissions. The extension and reconstruction of the volcanic forcing is related to a rescaling of the newly available Sigl et al. (2015) dataset to the reconstruction of Crowley and Unterman (2013). The large volcanoes for different latitudinal bands are rescaled according to 
https://doi.org/10.5194/cp-2022-5

Preprint. Discussion started: 31 January 2022

(c) Author(s) 2022. CC BY 4.0 License.

(c) (i)

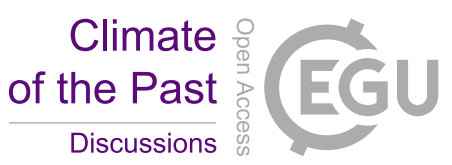

sulfate concentrations and eventually the Crowley algorithm was applied to yield aerosol optical depths and effective radius for four latitudinal bands separated by $30^{\circ}$.

\section{Appendix B}

Table B1. Skill reconstruction statistics for the North Hemisphere mean temperature in the verification period for noisecontaminated PPEs based on CCSM using different neuron numbers in hidden layers of Bi-LSTM method.

\begin{tabular}{ccc}
\hline Number of neurons & SD Ratio & cc \\
\cline { 2 - 3 } 50 neurons & 0.289 & 0.443 \\
200 neurons & 0.315 & 0.461 \\
500 neurons & 0.339 & 0.467 \\
700 neurons & 0.412 & 0.456 \\
\hline
\end{tabular}

In order to check whether the number of neurons in the hidden layer will substantial impact the CFR skills. We also conducted additional tests for our Bi-LSTM model structures with different neurons in the hidden layer based on one of the settings for PPEs. Table 1 indicates that, when we fix the rest of the hyper-parameters, etc. optimization function and learning rate, of the

485 Bi-LSTM structure, just tuning the number of neurons in the hidden layer does not yield a too strong impact on the mean correlation coefficients, but it results in an obvious impact on the SD ratio. Considering the variability capturing performance, it seems that the hidden layer with 700 neurons structure outperforms the hidden layer with less neurons structure, whereas the temporal covariance between target and reconstructed results does not change too much amongst these four different hidden layer structures. In our PPE tests on paleo CFRs, it seems that there is no specific neural network structure could universally outperform another one.

\section{Data availability}

The CCSM4 and MPI-ESM-P model output that was employed for this study is publicly accessible and can be downloaded by the Earth System Grid Federation (ESGF); https://esgf-data.dkrz.de/projects/esgf-dkrz/. The CESM1 model data can be downloaded: https://www.cesm.ucar.edu/projects/community-projects/LME/.

\section{Author contributions}

The analysis was performed by ZZ with the consultation of SW, MK and EZ. ZZ prepared the paper with contributions from all co-authors. 
https://doi.org/10.5194/cp-2022-5

Preprint. Discussion started: 31 January 2022

(c) Author(s) 2022. CC BY 4.0 License.

(c) (i)

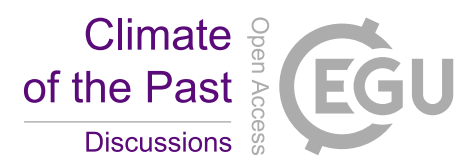

\section{Competing interests}

The authors declare that they have no conflict of interest.

\section{Acknowledgements}

The authors thank the CCSM4 and MPI-ESM modelling groups participating in the CMIP5 initiative for providing their data and the CESM1 modelling group for making their data available. This work is funded by the China Scholarship Council (no. 201806570017), and is part of the project Reduced Complexity Models (Redmod), funded by the Helmholtz Association through its Incubator program.

\section{References}

Amrhein, D. E., Hakim, G. J., and Parsons, L. A.: Quantifying structural uncertainty in paleoclimate data assimilation with an application to the Last Millennium, Geophys. Res. Lett., 47, e2020GL090485. https://doi.org/10.1029/2020GL090485, 2020. Anchukaitis, K. J., Wilson, R., Briffa, K. R., Büntgen, U., Cook, E. R., D’Arrigo, R., Davi, N., Esper, J., Frank, D., Gunnarson, B. E., Hegerl, G., Helama, S., Klesse, S., Krusic, P. J., Linderholm, H. W., Myglan, V., Osborn, T. J., Zhang, P., Rydval, M.,

510 Schneider, L., Schurer, A., Wiles, G., and Zorita, E.: Last millennium Northern Hemisphere summer temperatures from tree rings: Part II, spatially resolved reconstructions, Quaternary Sci. Rev., 163, 1-22, https://doi.org/10.1016/j.quascirev.2017.02.020, 2017.

Biswas, S., Sinha, M.: Performances of deep learning models for Indian Ocean wind speed prediction, Model. Earth Syst. Environ., 7, 809-831,https://doi.org/10.1007/s40808-020-00974-9, 2021.

515 Biswas, K., Kumar, S., and Pandey, A. K.: Intensity Prediction of Tropical Cyclones using Long Short-Term Memory Network. arXiv [preprint], https://arxiv.org/abs/2107.03187, 2021.

Christiansen, B. and Ljungqvist, F. C.: Challenges and perspectives for large-scale temperature reconstructions of the past two millennia, Rev. Geophys., 55, 40-96, https://doi.org/10.1002/2016RG000521, 2016.

Chattopadhyay, A., Hassanzadeh, P., and Subramanian, D.: Data-driven predictions of a multiscale Lorenz 96 chaotic system using machine-learning methods: reservoir computing, artificial neural network, and long short-term memory network, Nonlin. Processes Geophys., 27, 373-389, https://doi.org/10.5194/npg-27-373-2020, 2020.

Crowley, T. J. and Unterman, M. B.: Technical details concerning development of a $1200 \mathrm{yr}$ proxy index for global volcanism, Earth Syst. Sci. Data, 5, 187-197, https://doi.org/10.5194/essd-5-187-2013, 2013.

Cui, Z.; Ke, R.; Pu, Z.; Wang, Y. Deep bidirectional and unidirectional LSTM recurrent neural network for network-wide traffic speed prediction. arXiv [preprint], https://arxiv.org/abs/1801.02143, 2018.

Dueben, P. D. and Bauer, P.: Challenges and design choices for global weather and climate models based on machine learning, Geosci. Model Dev., 11, 3999-4009, https://doi.org/10.5194/gmd-11-3999-2018, 2018. 
https://doi.org/10.5194/cp-2022-5

Preprint. Discussion started: 31 January 2022

(c) Author(s) 2022. CC BY 4.0 License.

\section{(c) (1)}

Evans, M., Smerdon, J. E., Kaplan, A., Tolwinski-Ward, S., and González-Rouco, J. F.: Climate field reconstruction uncertainty arising from multivariate and nonlinear properties of predictors, Geophys. Res. Lett., 41, 9127-9134, https://doi.org/10.1002/2014g1062063, 2014.

Folland, C. K., Knight, J., Linderholm, H. W., Fereday, D., Ineson, S., and Hurrell, J. W.: The Summer North Atlantic Oscillation: Past, present, and future, J. Climate, 22(5), 1082-1103, doi:10.1175/2008JCLI2459.1, 2009.

Gao, C., Robock, A., and Ammann, C.: Volcanic forcing of climate over the past 1500 years: An improved ice core-based index for climate models, J. Geophys. Res.-Atmos., 113, D23111, https://doi.org/10.1029/2008JD010239, 2008.

535 Gómez-Navarro, J. J., Zorita, E., Raible, C. C., and Neukom, R.: Pseudo-proxy tests of the analogue method to reconstruct spatially resolved global temperature during the Common Era, Clim. Past, 13, 629-648, https://doi.org/10.5194/cp-13-6292017, 2017.

Graves, A., Schmidhuber, J.: Framewise phoneme classification with bidirectional LSTM and other neural and other neural network architectures, Neural Netw 18(5), 602-610. https://doi.org/10.1016/j.neunet.2005.06.042, 2005.

540 Gent, P. R., Danabasoglu, G., Donner, L. J., Holland, M. M., Hunke, E. C., Jayne, S. R., Lawrence, D. M., Neale, R. B., Rasch, P. J., and Vertenstein, M.: The community climate system model version 4, J. Clim., 24, 4973-4991, doi:10.1175/2011JCLI4083.1, 2011.

Goodfellow, I., Bengio, Y., and Courville, A.: Deep learning, MIT Press, 2016.

Guillot, D., Rajaratnam, B., and Emile-Geay, J.: Statistical paleoclimate reconstructions via Markov Random Fields, Ann. Appl. Stat., 9, 324-352, https://doi.org/10.1214/14-aoas794, 2015.

Hernández, A., Martin-Puertas, C., Moffa-Sánchez, P., Moreno-Chamarro, E., Ortega, P., Blockley, S., Cobb, K. M., ComasBru, L., Giralt, S., Goosse, H., Luterbacher, J., Martrat, B., Muscheler, R., Parnell, A., Pla-Rabes, S., Sjolte, J., Scaife, A. A., Swingedouw, D., Wise, E., and Xu, G.: Modes of climate variability: Synthesis and review of proxy-based reconstructions through the Holocene, Earth Sci. Rev., 271, 103286, https://doi.org/10.1016/j.earscirev.2020.103286, 2020.

Huntingford, C., Jeffers, E. S., Bonsall, M. B., Christensen, H. M., Lees, T., and Yang, H.: Machine learning and artificial intelligence to aid climate change research and preparedness, Environ. Res. Lett., 14, 124007, https://doi.org/10.1088/17489326/ab4e55, 2019.

Huang, Y., Yang, L., and Fu, Z.: Reconstructing coupled time series in climate systems using three kinds of machine-learning methods, Earth Syst. Dynam., 11, 835-853, https://doi.org/10.5194/esd-11-835-2020, 2020.

Hunke, E., Lipscomb, W., Turner, A., Jeffery, N., and Elliott, S.: CICE: the Los Alamos sea ice model, documentation and software, version 4.0. Los Alamos National Laboratory, Tech. Rep, LA-CC-06-012, 2008. Los Alamos National Laboratory, Los Alamos, NM.

Hurrell, J. W., Holland, M. M., Gent, P. R., Ghan, S., Kay, J. E., Kushner, P. J., Lamarque, J. F., Large, W. G., Lawrence, D., Lindsay, K., Lipscomb, W. H., Long, M. C., Mahowald, N., Marsh, D. R., Neale, R. B., Rasch, P., Vavrus, S., Vertenstein, M., Bader, D., Collins, W. D., Hack, J. J., Kiehl, J., and Marshall, S.: The Community Earth System Model: A Framework for Collaborative Research, B. Am. Meteorol. Soc., 94, 1339-1360, https://doi.org/10.1175/bams-d-12-00121.1, 2013. 
https://doi.org/10.5194/cp-2022-5

Preprint. Discussion started: 31 January 2022

(c) Author(s) 2022. CC BY 4.0 License.

\section{(c) (1)}

Hurtt, G. C., Chini, L. P., Frolking, S., Betts, R., Feddema, J., Fischer, G., Goldewijk, K. K., Hibbard, K., Janetos, A., and Jones, C.: Harmonisation of global land-use scenarios for the period 1500-2100 for IPCC-AR5, ILEAPS Newsletter, No. 7, 6-8, 2009.

565 Hotelling, H.: The relations of the newer multivariate statistical methods to factor analysis, Brit. J. Statist. Psych., 10, 69-76, https://doi.org/10.1111/j.2044-8317.1957.tb00179.x, 1957.

Hodges, J. L.: The significance probability of the Smirnov two-sample test, Arkiv för Matematik, 3, 469-486, 1958.

Ilyina, T., Six, K. D., Segschneider, J., Maier-Reimer, E., Li, H., and Núñez-Riboni, I.: Global ocean biogeochemistry model HAMOCC: Model architecture and performance as component of the MPI-Earth system model in different CMIP5 experimental realizations, J. Adv. Model. Earth Syst., 5, 287-315, 2013.

Jacobeit, J., Wanner, H., Luterbacher, J., Beck, C., Philipp, A., and Sturm, K.: Atmospheric circulation variability in the NorthAtlantic-European area since the mid-seventeenth century, Clim. Dynam., 20, 341-352, https://doi.org/10.1007/s00382002-0278-0, 2003.

Jahangir, H., Tayarani, H., Gougheri, S.S., Golkar, M.A., Ahmadian, A., Elkamel, A.: Deep Learning-based Forecasting Approach in Smart Grids with Micro-Clustering and Bi-directional LSTM Network. IEEE Trans. Ind. Electron., 68, 82988309, doi: 10.1109/TIE.2020.3009604, 2020.

Jungclaus, J. H., Fischer, N., Haak, H., Lohmann, K., Marotzke, J., Matei, D., Mikolajewicz, U., Notz, D., and vonStorch, J. S.: Characteristics of the ocean simulations in MPIOM, the ocean component of the MPI-Earth system model, J. Adv. Model. Earth Syst., 5, 422-446, doi:10.1002/jame.20023, 2013.

580 Kingma, D., Ba, J.: Adam: a method for stochastic optimization. arXiv [preprint], https://arxiv.org/abs/1412.6980, 2014.

Knight, J. R., Folland, C. K., and Scaife, A. A.: Climate impacts of the Atlantic Multidecadal Oscillation, Geophys. Res. Lett., 33, L17706, doi:10.1029/2006GL026242, 2006.

Kadow, C., Hall, D. M., and Ulbrich, U.: Artificial intelligence reconstructs missing climate information, Nat. Geosci., 13, 408-413, https://doi.org/10.1038/s41561-020-0582-5, 2020.

585 Lean, J., Rottman, G., Harder, J., and Kopp, G.: SORCE contributions to new understanding of global change and solar variability, in: The Solar Radiation and Climate Experiment (SORCE), Springer, 2005.

Luterbacher, J., Dietrich, D., Xoplaki, E., Grosjean, M., and Wanner, H.: European seasonal and annual temperature variability, trends, and extremes since 1500, Science, 303, 1499-1503, DOI: 10.1126/science.1093877, 2004.

Luterbacher J., Werner, J. P., Smerdon, J. E., Fernández-Donado, L., González-Rouco, F. J., Barriopedro, D., Ljungqvist, F. C., Büntgen, U., Zorita, E., Wagner, S., Esper, J., McCarroll, D., Toreti, A., Frank, D., Jungclaus, J. H., Barriendos, M., Bertolin, C., Bothe, O., Brázdil, R., Camuffo, D., Dobrovolný, P., Gagen, M., García-Bustamante, E., Ge, Q., Gómez-Navarro, J. J., Guiot, J., Hao, Z., Hegerl, G. C., Holmgren, K., Klimenko, V. V., MartínChivelet, J., Pfister, C., Roberts, N., Schindler, A., Schurer, A., Solomina, O., von Gunten, L., Wahl, E., Wanner, H., Wetter, O., Xoplaki, E., Yuan, N., Zanchettin, D., Zhang, H., and Zerefos, C.: European summer temperatures since Roman times, Environ. Res. Lett., 11, 024001, doi:10.1088/17489326/11/2/024001, 2016. 
https://doi.org/10.5194/cp-2022-5

Preprint. Discussion started: 31 January 2022

(c) Author(s) 2022. CC BY 4.0 License.

(c) (i)

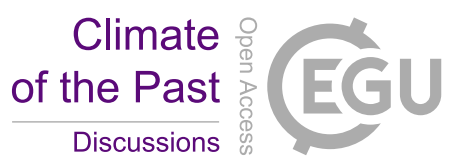

Lindgren, A., Lu, Z., Zhang, Q., and Hugelius, G.: Reconstructing past global vegetation with random forest machine learning, sacrificing the dynamic response for robust results J. Adv. Model. Earth. Syst., 13, p.e2020MS002200. https://doi.org/10.1029/2020MS002200, 2021.

Lawrence, D. M., Oleson, K. W., Flanner, M. G., Fletcher, C. G., Lawrence, P. J., Levis, S., Swenson, S. C., and Bonan, G.

B.: The CCSM4 land simulation, 1850-2005: Assessment of surface climate and new capabilities, J. Clim., 25, 2240-2260, doi:10.1175/JCLI-D-11-00103.1, 2012.

Li, B. and Smerdon, J. E.: Defining spatial comparison metrics for evaluation of paleoclimatic field reconstructions of the Common Era, Environmetrics, 23, 394-406, doi:10.1002/env.2142, 2012.

Mann, M. E., Bradley, R. S., and Hughes, M. K.: Global-scale temperature patterns and climate forcing over the past six 605 centuries, Nature, 392, 779-787, https://doi.org/10.1038/33859, 1998.

Mann, M. E. and Rutherford, S.: Climate reconstruction using "Pseudoproxies", Geophys. Res. Lett., 29, 1501, https://doi.org/10.1029/2001GL014554, 2002.

Mann, M. E., Rutherford, S., Wahl, E., and Ammann, C.: Testing the fidelity of methods used in proxy-based reconstructions of past climate, J. Clim., 18, 4097-4107, https://doi.org/10.1175/JCLI3564.1, 2005.

610 Mann, M. E., Rutherford, S., Wahl, E., and Ammann, C.: Robustness of proxy-based climate field reconstruction methods, J. Geophys. Res.-Atmos., 112, D12109, doi:10.1029/2006JD008272, 2007.

Mann, M. E., Zhang, Z., Hughes, M. K., Bradley, R. S., Miller, S. K., Rutherford, S., and Ni, F.: Proxy-based reconstructions of hemispheric and global surface temperature variations over the past two millennia, P. Natl. A. Sci., 105(36), 13252-13257, https://doi.org/10.1073/pnas.0805721105, 2008.

615 Mann, M. E., Zhang, Z., Rutherford, S., Bradley, R. S., Hughes, M. K., Shindell, D., Ammann, C., Faluvegi, G., and Ni, F.: Global signatures and dynamical origins of the Little Ice Age and Medieval Climate Anomaly, Science, 326, 1256-1260, DOI: 10.1126/science.1177303, 2009a.

Mann, M. E., Woodruff, J. D., Donnelly, J. P., and Zhang, Z.: Atlantic hurricanes and climate over the past 1,500 yr, Nature, 460, 880-883, doi:10.1038/nature08219, 2009b.

620 Meyer, G.P.: An Alternative Probabilistic Interpretation of the Huber Loss. arXiv [preprint], https://arxiv.org/abs/1911.02088, 2020.

Michel, S., Swingedouw, D., Chavent, M., Ortega, P., Mignot, J., and Khodri, M.: Reconstructing climatic modes of variability from proxy records using ClimIndRec version 1.0, Geosci. Model Dev., 13, 841-858, https://doi.org/10.5194/gmd-13-8412020, 2020.

625 Nadiga, B.: Reservoir Computing as a Tool for Climate Predictability Studies, J. Adv. Model. Earth. Syst., p. e2020MS002290, https://doi.org/10.1029/2020MS002290, 2020.

Neale, R. B., Richter, J., Park, S., Lauritzen, P. H., Vavrus, S. J., Rasch, P. J., and Zhang, M.: The mean climate of the Community Atmosphere Model (CAM4) in forced SST and fully coupled experiments, J. Clim., 26, 5150-5168, doi:10.1175/JCLI-D-12-00236.1, 2013. 
https://doi.org/10.5194/cp-2022-5

Preprint. Discussion started: 31 January 2022

(c) Author(s) 2022. CC BY 4.0 License.

(c) (i)

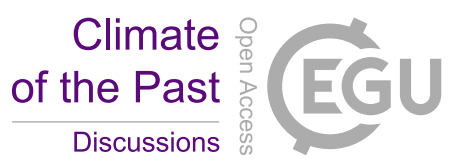

630 Otto-Bliesner, B. L., Brady, E. C., Fasullo, J., Jahn, A., Landrum, L., Stevenson, S., Rosenbloom, N., Mai, A., and Strand, G.: CLIMATE VARIABILITY AND CHANGE SINCE 850 CE An Ensemble Approach with the Community Earth System Model, B. Am. Meteorol. Soc., 97, 735-754, https://doi.org/10.1175/bamsd-14-00233.1, 2016.

PAGES 2k Consortium: Continental-scale temperature variability during the last two millennia, Nat. Geosci., 6, 339-346, doi:10.1038/ngeo1797, 2013.

635 PAGES 2k Consortium: A global multiproxy database for temperature reconstructions of the Common Era, Sci. Data, 4, 170088, https://doi.org/10.1038/sdata.2017.88, 2017.

PAGES 2k Consortium: Consistent multidecadal variability in global temperature reconstructions and simulations over the Common Era, Nat. Geosci., 536, 411, https://doi.org/10.1038/s41561-019-0400-0, 2019.

PAGES Hydro2k Consortium: Comparing proxy and model estimates of hydroclimate variability and change over the 640 Common Era, Clim. Past, 13, 1851-1900, https://doi.org/10.5194/cp-13-1851-2017, 2017.

Pongratz, J., Reick, C., Raddatz, T., and Claussen, M.: A reconstruction of global agricultural areas and land cover for the last millennium, Global Biogeochem. Cy., 22, GB3018, https://doi.org/10.1029/2007GB003153, 2008.

Po-Chedley, S., Santer, B. D., Fueglistaler, S., Zelinka, M., Cameron-Smith, P., Painter, J., and Fu, Q.: Natural variability contributes to model-satellite differences in tropical tropospheric warming. Proc. Natl Acad. Sci., 118(13), e2020962118, 645 https://doi.org/10.1073/pnas.2020962118, 2020.

Ramachandran P., Zoph B., and Le QV.: Searching for activation functions. arXiv [preprint], https://arxiv.org/abs/1710.05941, 2017.

Pyrina, M., Wagner, S., and Zorita, E.: Pseudo-proxy evaluation of climate field reconstruction methods of North Atlantic climate based on an annually resolved marine proxy network, Clim. Past, 13, 1339-1354, https://doi.org/10.5194/cp-13-1339-

$650 \quad 2017,2017$.

Qasmi, S., Cassou, C., and Boé, J.: Teleconnection Between Atlantic Multidecadal Variability and European Temperature: Diversity and Evaluation of the Coupled Model Intercomparison Project Phase 5 Models, Geophys. Res. Lett., 44, 11-140, https://doi.org/10.1002/2017GL074886, 2017.

Rasp, S. and Lerch, S.: Neural Networks for Postprocessing Ensemble Weather Forecasts, Mon. Weather Rev., 146, 3885655 3900, https://doi.org/10.1175/MWR-D-18-0187.1, 2018.

Rolnick, D., Donti, P. L., Kaack, L. H., Kochanski, K., Lacoste, A., Sankaran, K., Ross, A. S., Milojevic-Dupont, N., Jaques, N., Waldman-Brown, A., Luccioni, A., Maharaj, T., Sherwin, E. D., Mukkavilli, S. K., Kording, K. P., Gomes, C., Ng, A. Y., Hassabis, D., Platt, J. C., Creutzig, F., Chayes, J., and Bengio, Y.: Tackling climate change with machine learning, arXiv [preprint], arXiv:1906.05433. https://arxiv.org/abs/1906.05433, 2019.

660 Reick, C. H., Raddatz, T., Brovkin, V., and Gayler, V.: Representation of natural and anthropogenic land cover change in MPIESM, J. Adv. Model. Earth Syst., 5, 459-482, doi:10.1002/jame.20022, 2013.

Schmidt, G. A.: Enhancing the relevance of paleoclimatic model/data comparisons for assessments of future climate change, J Quaternary Sci., 25, 79-87, doi:10.1002/jqs.1314, 2010. 
https://doi.org/10.5194/cp-2022-5

Preprint. Discussion started: 31 January 2022

(c) Author(s) 2022. CC BY 4.0 License.

(c) (i)

Schmidt, G. A., Jungclaus, J. H., Ammann, C. M., Bard, E., Braconnot, P., Crowley, T. J., Delaygue, G., Joos, F., Krivova, N.

A., Muscheler, R., Otto-Bliesner, B. L., Pongratz, J., Shindell, D. T., Solanki, S. K., Steinhilber, F., and Vieira, L. E. A.:

Climate forcing reconstructions for use in PMIP simulations of the last millennium (v1.0), Geosci. Model Dev., 4, 33-45, https://doi.org/10.5194/gmd-4-33-2011, 2011.

Smerdon, J. E.: Climate models as a test bed for climate reconstruction methods: pseudoproxy experiments, Wiley Interdisciplinary Reviews, Clim. Change., 3, 63-77, https://doi.org/10.1002/wcc.149, 2012.

Smerdon, J. E., Kaplan, A., Zorita, E., Gonzalez-Rouco, J. F., and Evans, M. N.: Spatial performance of four climate field reconstruction methods targeting the Common Era, Geophys. Res. Lett., 38, L11705, doi:10.1029/2011GL047372, 2011.

Smerdon, J. E., Coats, S., and Ault, T. R.: Model-dependent spatial skill in pseudoproxy experiments testing climate field reconstruction methods for the Common Era, Clim. Dynam., 46, 1921-1942, https://doi.org/10.1007/s00382-015-2684-0, 2016.

675 Smith, R., Jones, P., Briegleb, B., Bryan, F., Danabasoglu, G., Dennis,J., Dukowicz, J., Eden, C., Fox-Kemper, B., and Gent, P.: The Parallel Ocean Program (POP) Reference Manual Ocean Component of the Community Climate System Model (CCSM) and Community Earth System Model (CESM), Rep. LAUR-01853, 1-141, 2010.

Sutton, R. T. and Hodson, D. L. R.: Atlantic ocean forcing of North American and European summer climate, Science, 309, 115- 118, doi:10.1126/science.1109496, 2005.

680 St. George, S.: An overview of tree-ring width records across the Northern Hemisphere, Quaternary Sci. Rev., 95, 132-150, https://doi.org/10.1016/j.quascirev.2014.04.029, 2014.

St. George, S. and Esper, J.: Concord and discord among Northern Hemisphere paleotemperature reconstructions from tree rings, Quat. Sci. Rev., 203, 278-281, https://doi.org/10.1016/j.quascirev.2018.11.013, 2019.

Schneider, T., Lan, S., Stuart, A., and Teixeira, J.: Earth System Modeling 2.0: A Blueprint for Models That Learn From

Observations and Targeted High-Resolution Simulations, Geophys. Res. Lett., 44, 12396-12417, https://doi.org/10.1002/2017GL076101, 2018.

Su, H., Zhang, T., Lin, M., Lu, W., Yan, X. H.: Predicting subsurface thermohaline structure from remote sensing data based on long short-term memory neural networks, Remote Sens. Environ., 260, 112465 10.1016/j.rse.2021.112465, 2021.

Steiger, N. J., Hakim, G. J., Steig, E. J., Battisti, D. S., and Roe, G. H.: Assimilation of time-averaged pseudoproxies for climate reconstruction, J. Clim., 27, 426-441, https://doi.org/10.1175/JCLI-D-12-00693.1, 2014.

Stevens, B., Giorgetta, M., Esch, M., Mauritsen, T., Crueger, T., Rast, S., Salzmann, M., Schmidt, H., Bader, J., Block, K., Brokopf, R., Fast, I., Kinne, S., Kornblueh, L., Lohmann, U., Pincus, R., Reichler, T., and Roeckner, E.: Atmospheric component of the MPI-M Earth System Model: ECHAM6-HAM2, J. Adv. Model. Earth Syst., 5, 146-172, doi:10.1002/jame.20015, 2013.

695 Sigl, M., Winstrup, M., McConnell, J. R., Welten, K C., Plunkett, G., Ludlow, F., Büntgen, U., Caffee, M., Chellman, N., Dahl-Jensen, D., Fischer, H., Kipfstuhl, S., Kostick, C., Maselli, O. J., Mekhaldi, F., Mulvaney, R., Muscheler, R., Pasteris, 
https://doi.org/10.5194/cp-2022-5

Preprint. Discussion started: 31 January 2022

(c) Author(s) 2022. CC BY 4.0 License.

(c) (i)

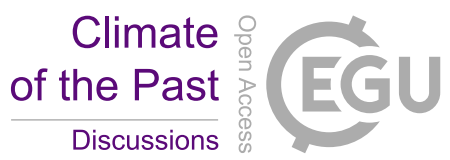

D. R., Pilcher, J. R., Salzer, M., Schüpbach, S., Steffensen, J. P., Vinther, B. M., and Woodruff, T. E.: Timing and climate forcing of volcanic eruptions for the past 2,500 years, Nature, 523, 543-549, doi:10.1038/nature14565, 2015.

von Storch, H., Zorita, E., Jones, J. M., Dimitriev, Y., González-Rouco, F., and Tett, S. F.: Reconstructing past climate from noisy data, Science, 306, 679-682, DOI: 10.1126/science.1096109, 2004.

Vieira, L. E. A., Solanki, S. K., Krivova, N. A., and Usoskin, I.: Evolution of the solar irradiance during the Holocene, Astron. Astrophys., 531, A6, doi:10.1051/0004-6361/201015843, 2011.

Wang, J., Emile-Geay, J., Guillot, D., Smerdon, J. E., and Rajaratnam, B. : Evaluating climate field reconstruction techniques using improved emulations of real-world conditions, Clim. Past, 10, 1-19, https://doi.org/10.5194/cp-10-1-2014, 2014.

705 Wang, J., Yang, B., Ljungqvist, F. C., Luterbacher, J., Osborn, T. J., Briffa, K. R., and Zorita, E.: Internal and external forcing of multidecadal Atlantic climate variability over the past 1,200 years, Nat. Geosci., 10, 512-517, https://doi.org/10.1038/ngeo2962, 2017.

Wilson, R., Anchukaitis, K., Briffa, K. R., Buentgen, U., Cook, E., D’Arrigo, R., Davi, N., Esper, J., Frank, D., Gunnarson, B., Hegerl, G., Helama, S., Klesse, S., Krusic, P. J., Linderholm, H. W., Myglan, V., Osborn, T. J., Rydval, M., Schneider, L.,

710 Schurer, A., Wiles, G., Zhang, P., and Zorita, E.: Last millennium Northern Hemisphere summer temperatures from tree rings: Part I: The long term context, Quaternary Sci. Rev., 134, 1-18, https://doi.org/10.1016/j.quascirev.2015.12.005, 2016.

Widmann, M.: One-Dimensional CCA and SVD, and Their Relationship to Regression Maps, J. Climate, 18, 2785-2792, https://doi.org/10.1175/jcli3424.1, 2005.

Wagner, S. and Zorita, E.: The influence of volcanic, solar and CO2 forcing on the temperatures in the Dalton Minimum 715 (1790-1830): a model study, Clim. Dynam., 25, 205-218, doi:10.1007/s00382-005-0029-0, 2005.

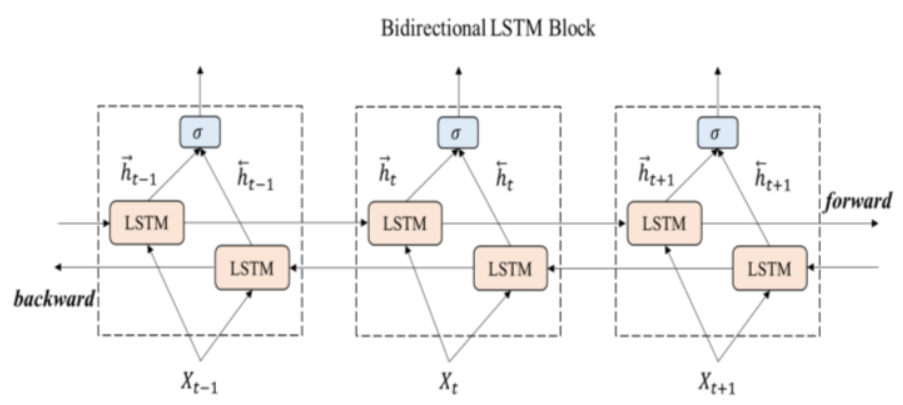

Figure 1: the bidirectional structure of the Bi-LSTM network. 

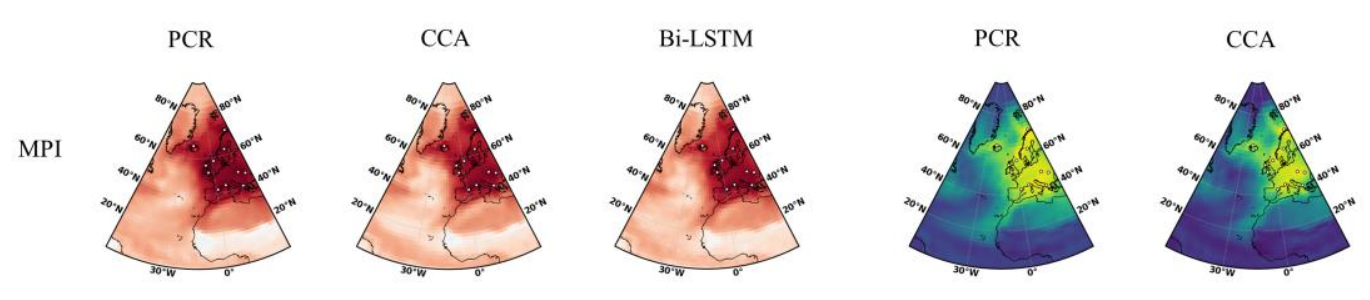

Bi-LSTM
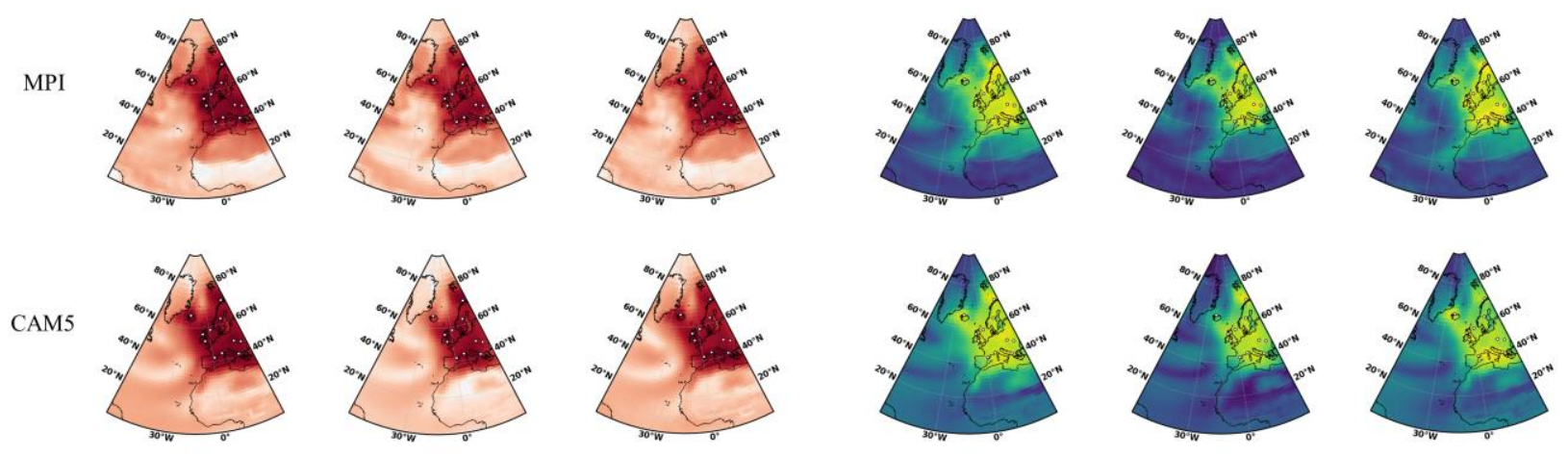

ccss
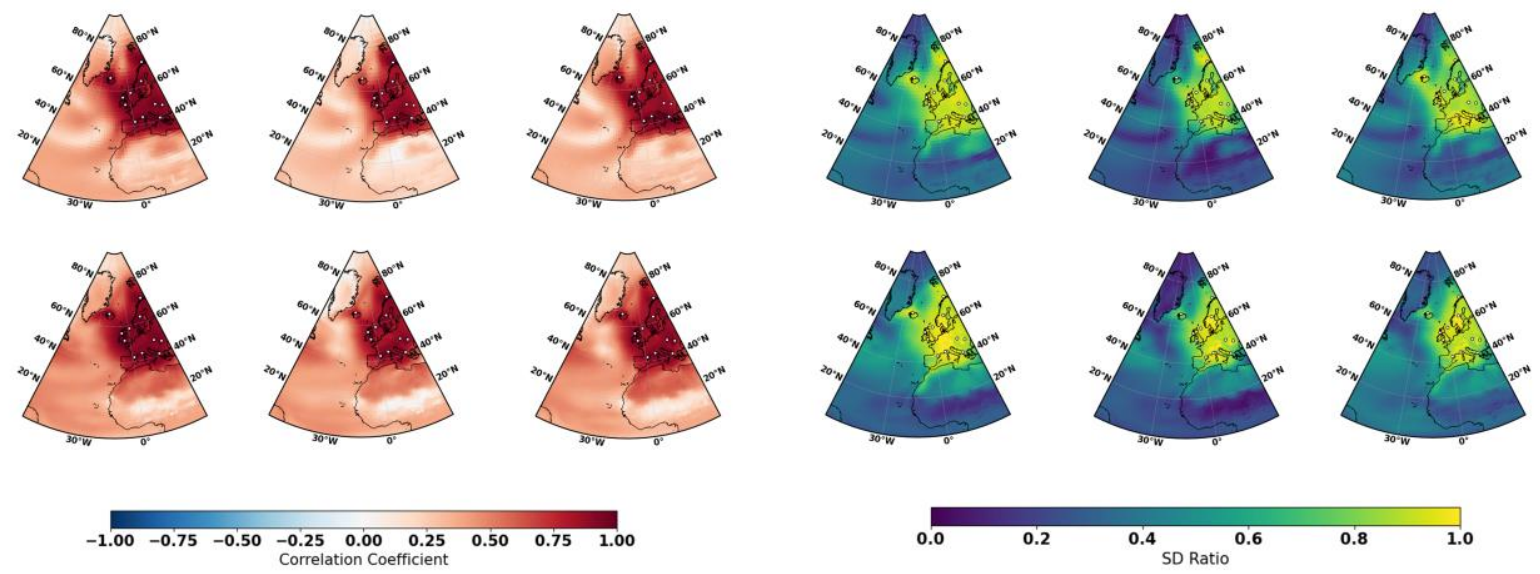

720

Figure 2: NAE Reconstruction results of CFR methods (including PCR, CCA, Bi-LTSM : Bidirectional long short term memory neural networks) using MPI, CESM1-CAM5 and CCSM numerical simulation as target temperature field, all the CFR methods employ the same proxy network with full 11 ideal pseudoproxies which span the same reconstruction period from 1426-2000 AD. The employed pseudoproxies geolocations are show in white circles in all the sub-figures ; CC is Correlation

725 Coefficient and SD represents Standard Deviation Ratio. The employed pseudoproxies' geolocation is shown as white circles in all the sub-figures. 

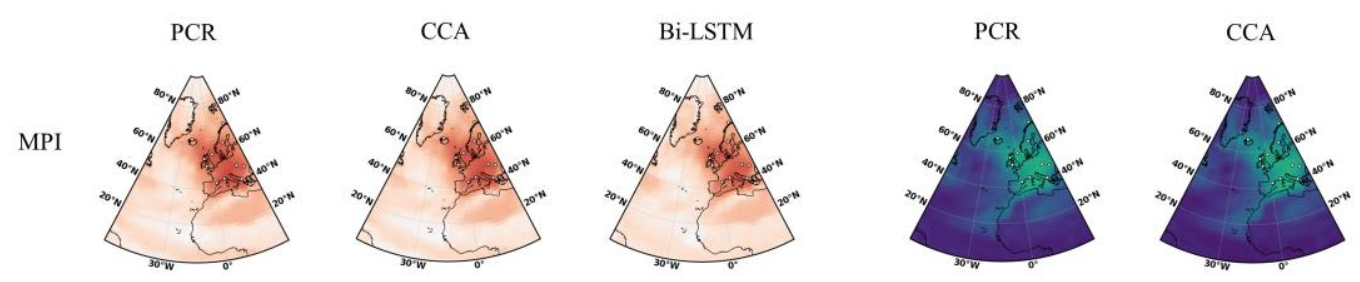

Bi-LSTM
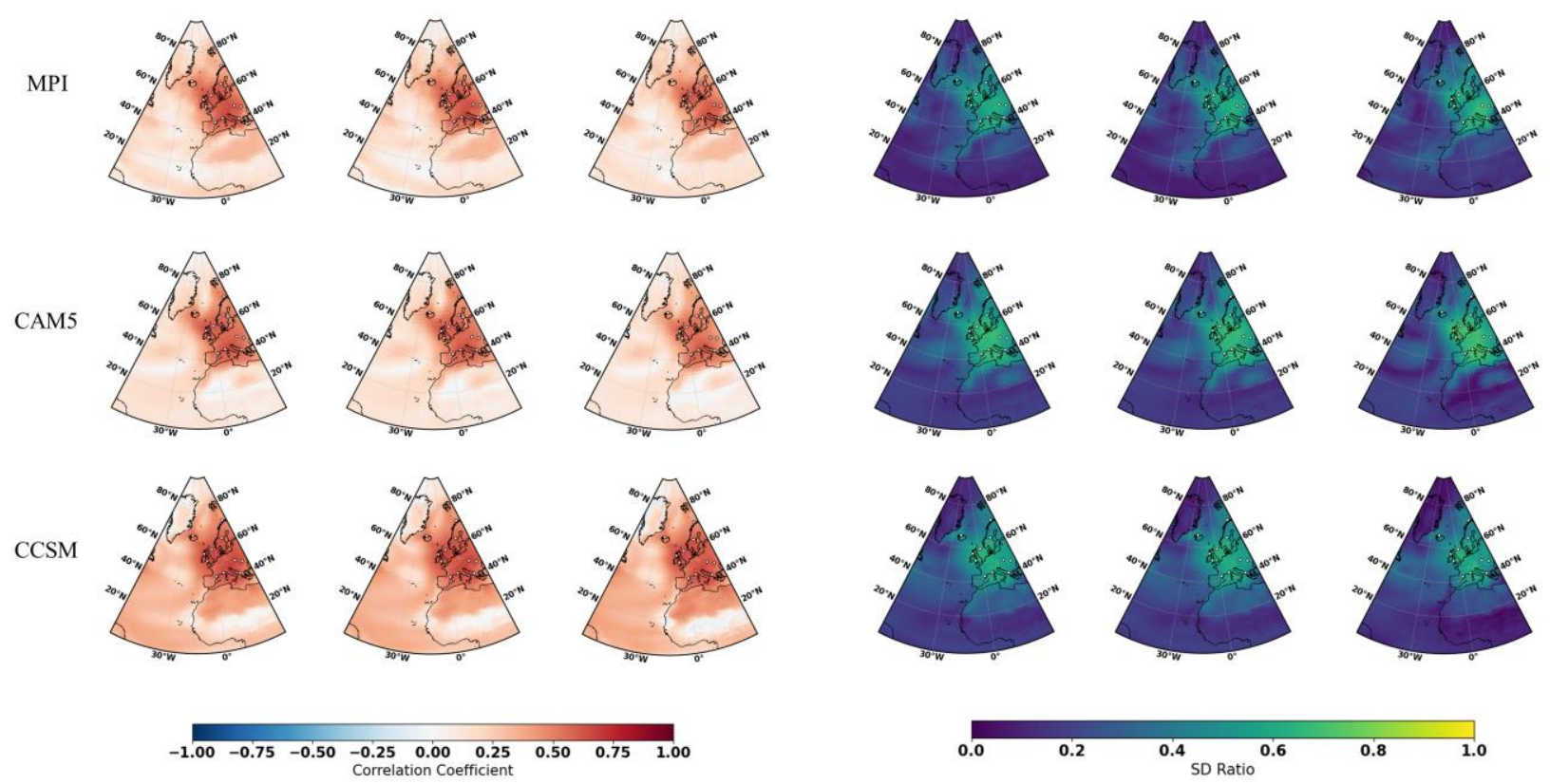

Figure 3: the same as Figure 2, but for employing the full 11 pseudoproxies network with 80\% noise contamination. 
https://doi.org/10.5194/cp-2022-5

Preprint. Discussion started: 31 January 2022

(c) Author(s) 2022. CC BY 4.0 License.

(c) (1)
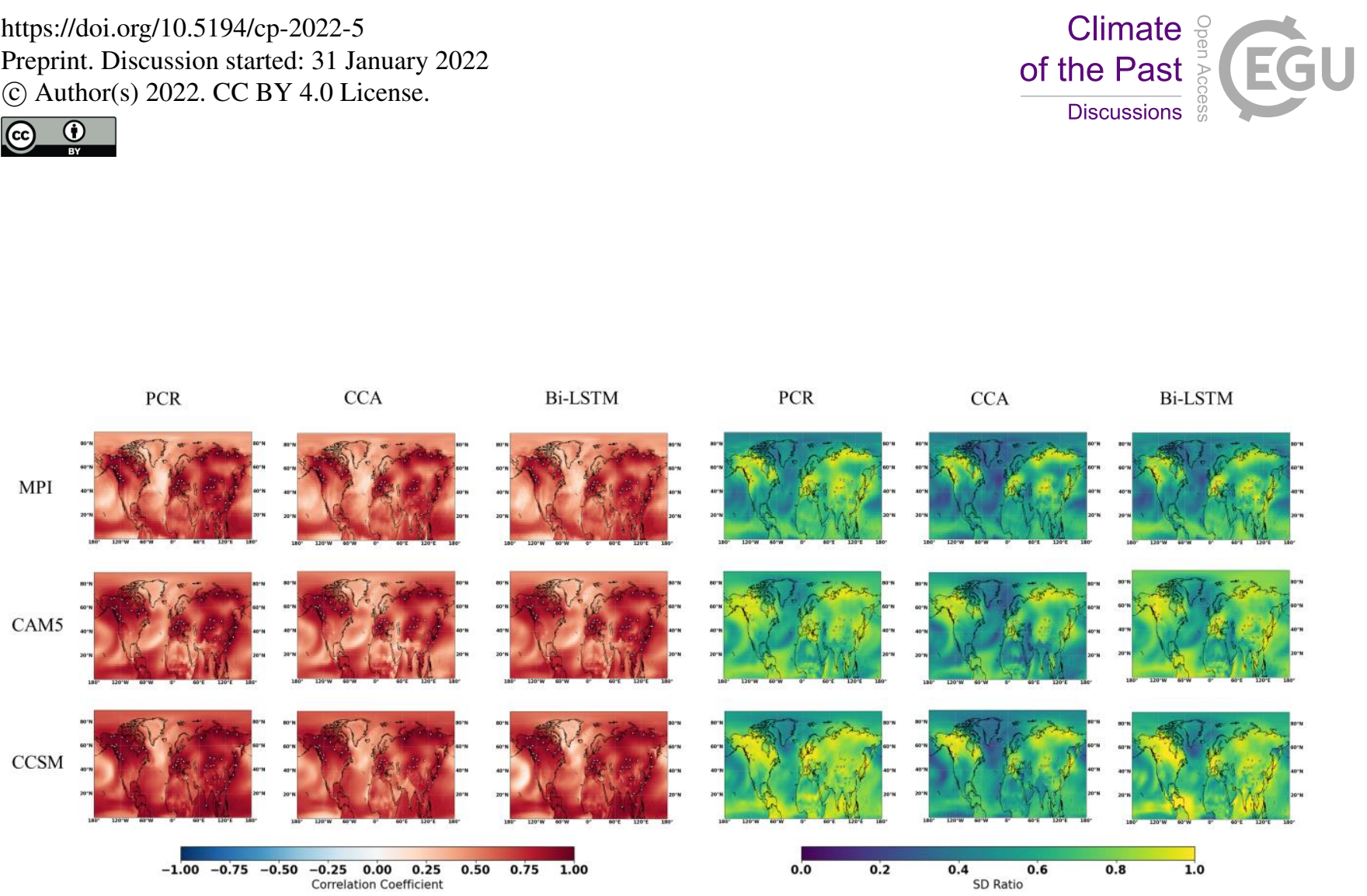

730

Figure 4: NH Reconstruction results of CFR methods (including PCR, CCA, Bi-LSTM: Bidirectional long short term memory neural networks) using MPI, CESM1-CAM5 and CCSM numerical simulation as target temperature field, all the CFR methods employ the same proxy network with full 48 ideal pseudoproxies which span the same reconstruction period from 1426-2000 AD. The employed pseudoproxies geolocations based on TRW are shown in white circles in all the sub-figures; CC is 735 Correlation Coefficient and SD represents Standard Deviation Ratio. The employed pseudoproxies' geolocation is shown as white circles in all the sub-figures . 
https://doi.org/10.5194/cp-2022-5

Preprint. Discussion started: 31 January 2022

(c) Author(s) 2022. CC BY 4.0 License.

(c) (i)

\section{Climate of the Past \\ Discussions

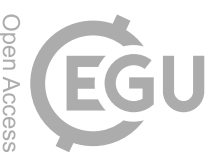

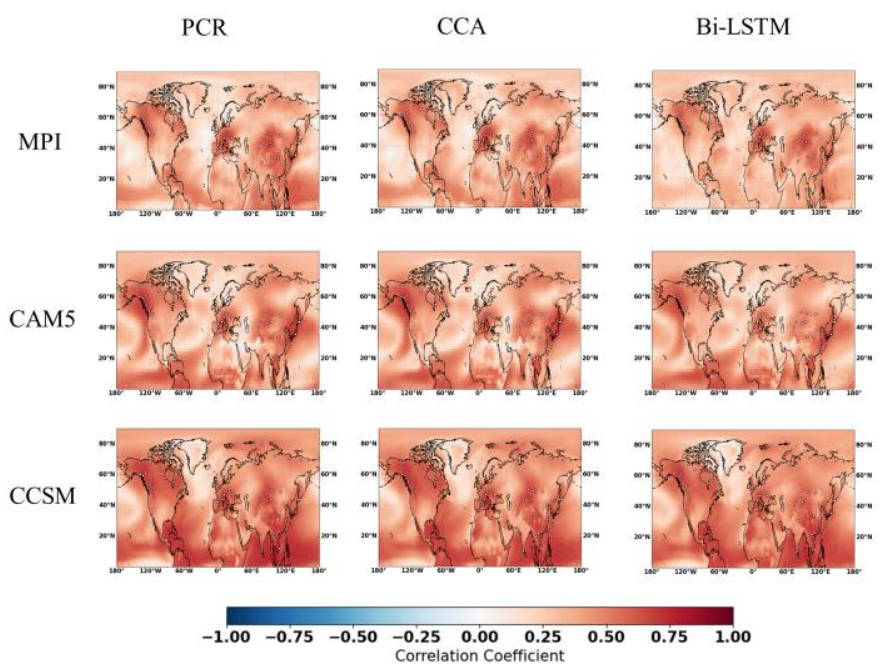
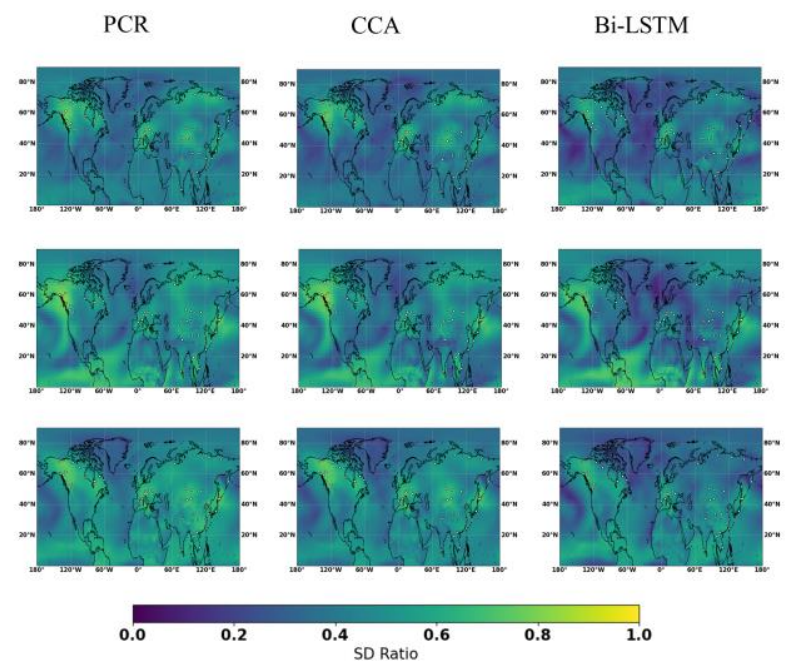

Figure 5: the same as Figure 4, but for employing the full 48 pseudoproxies network with $80 \%$ noise contamination. 


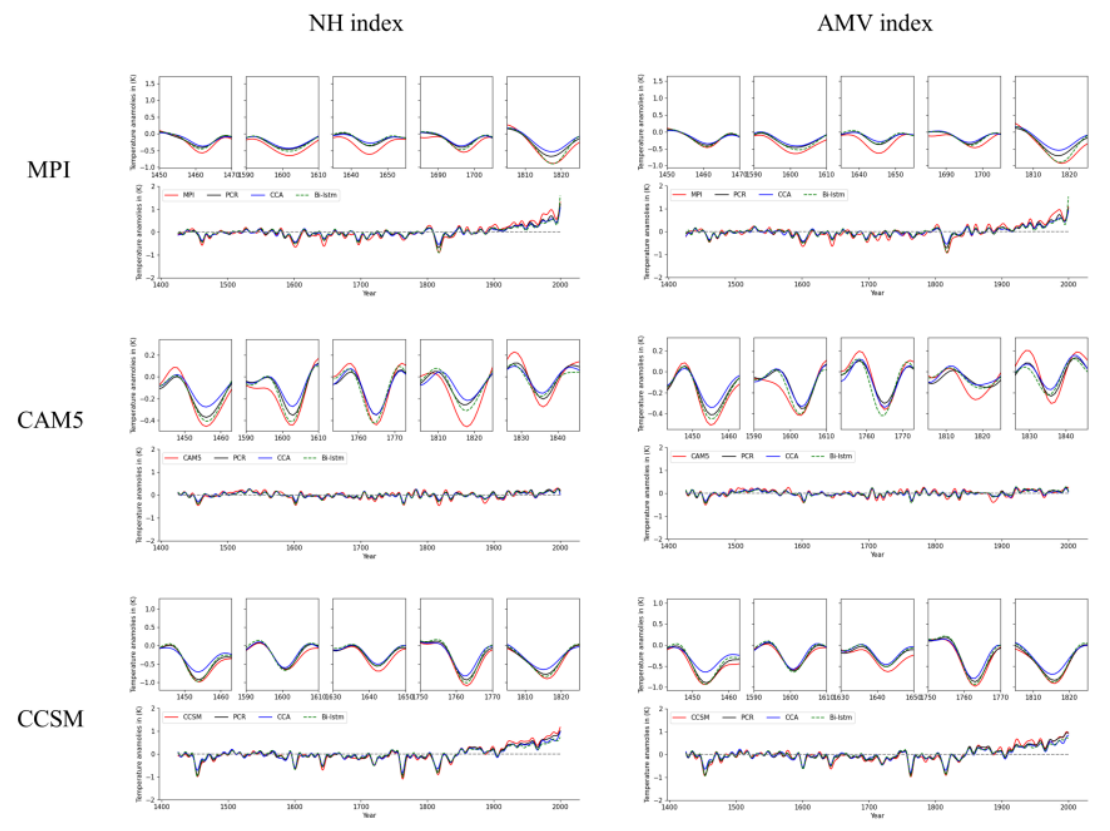

Figure 6: mean time series evolution of the validated reconstructions for $\mathrm{NH}$ summer temperature anomaly and Atlantic Multidecadal Variability (AMV) index using full 48 ideal-pseudoproxies based on PCR, CCA, Bi-LSTM CFR methods. All time series have been smoothed using a Butterworth low-pass filter to remove temporal fluctuations less than 10 years. MPI, CAM5 and CCSM represent MPI/CAM5/CCSM model simulated 'true' climatology. We selected several reconstructed extreme cooling period with a shorter interval (each 10 years are selected before and after the specific extreme cooling year) and plotted them above each entire reconstruction means amongst models and methods. 
NH index
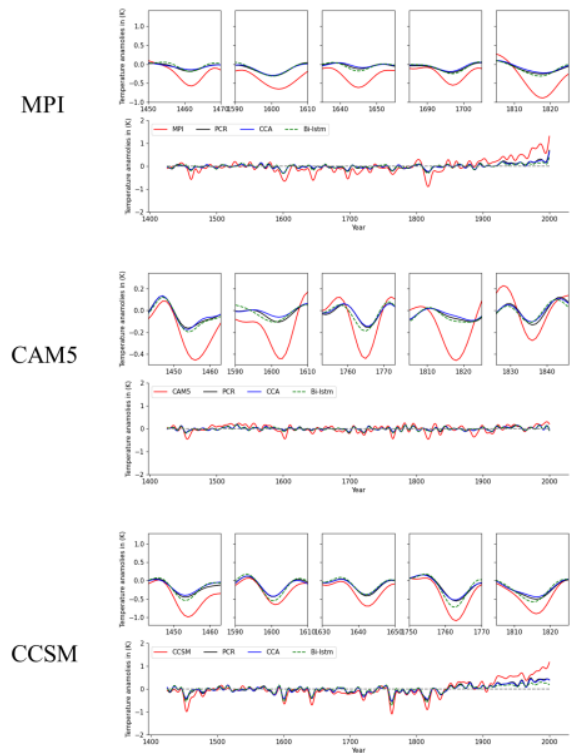

AMV index
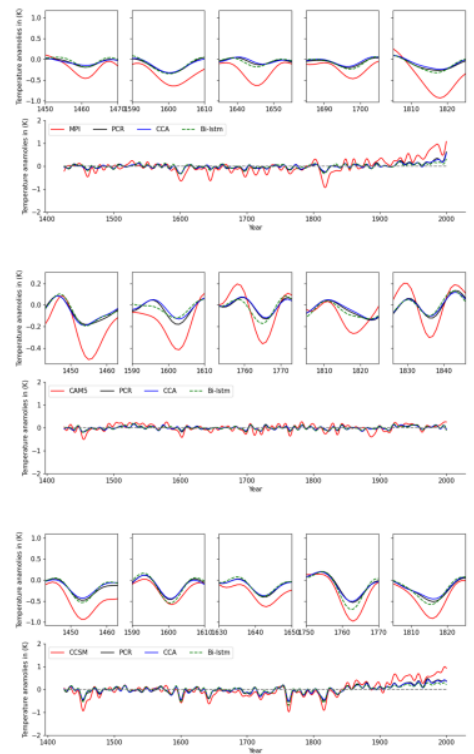

Figure 7: The same as Figure 6, but derived from $80 \%$ noise contaminated PPEs.
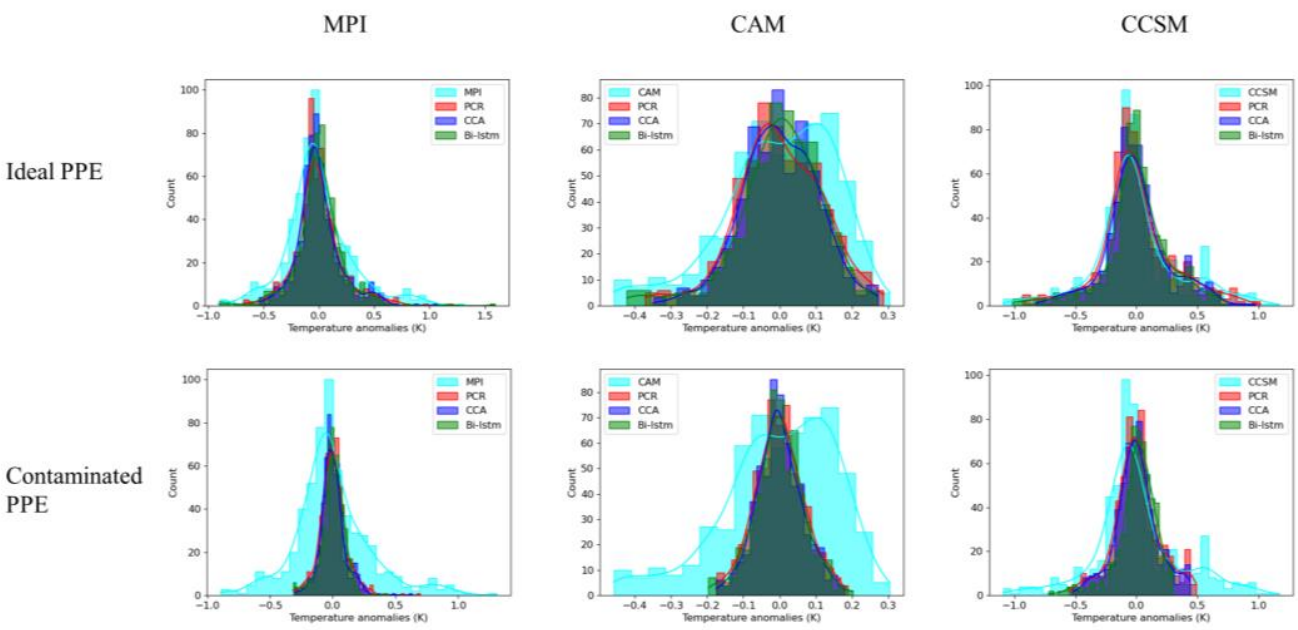

Figure 8: Histogram for decadal filtered NH mean index. The $\mathrm{x}$ axis denotes temperature anomaly values, and $\mathrm{y}$ axis is the number of data in each bin. Totally 30 bins are selected to plot each of the histogram. 
https://doi.org/10.5194/cp-2022-5

Preprint. Discussion started: 31 January 2022

(c) Author(s) 2022. CC BY 4.0 License.

(c) (i)
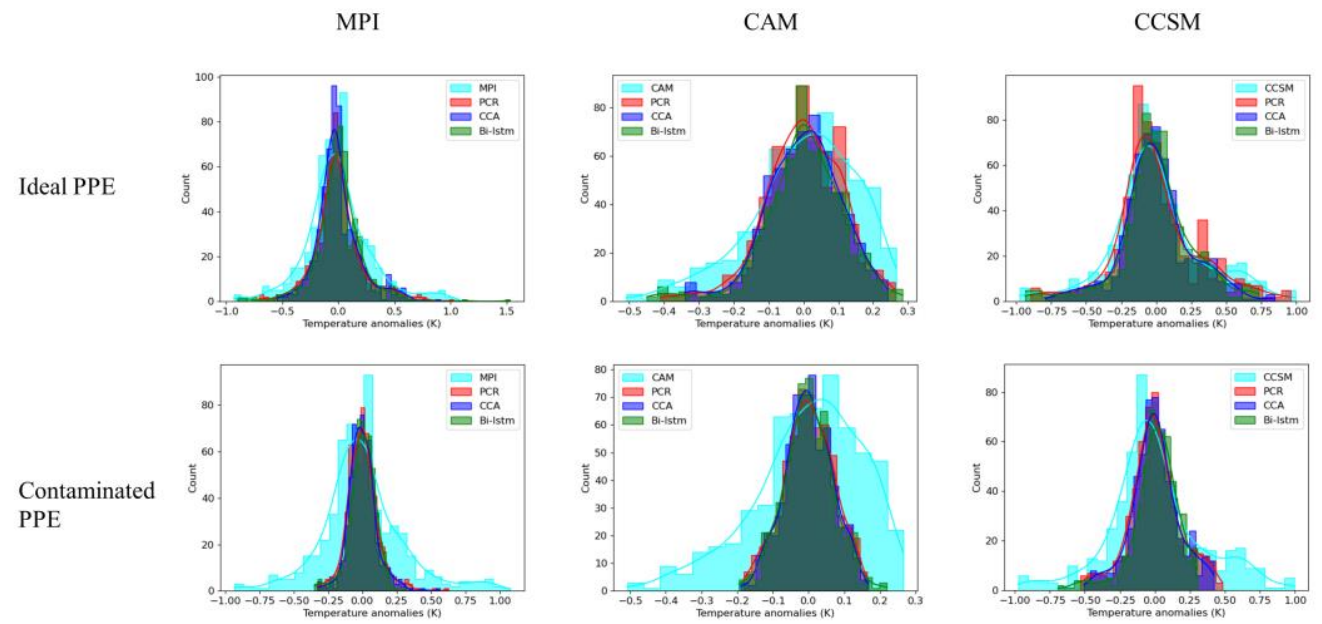

Figure 9: The same as Figure 8, but for decadal filtered AMV index. 